\title{
Three-dimensional numerical general relativistic hydrodynamics. II. Long-term dynamics of single relativistic stars
}

\author{
José A. Font, ${ }^{1,2}$ Tom Goodale, ${ }^{3}$ Sai Iyer, ${ }^{4}$ Mark Miller ${ }^{4}$ Luciano Rezzolla,${ }^{5,6}$ Edward Seidel,,${ }^{3,7}$ Nikolaos Stergioulas, ${ }^{8}$ \\ Wai-Mo Suen, ${ }^{4,9}$ and Malcolm Tobias ${ }^{4}$ \\ ${ }^{1}$ Max-Planck-Institut für Astrophysik, Karl-Schwarzschild-Str. 1, D-85740 Garching, Germany \\ ${ }^{2}$ Departamento de Astronomía y Astrofísica, Universidad de Valencia, 46100 Burjassot (Valencia), Spain \\ ${ }^{3}$ Max-Planck-Institut für Gravitationsphysik, Am Mühlenberg 1, D-14476 Golm, Germany \\ ${ }^{4}$ McDonnell Center for the Space Sciences, Department of Physics, Washington University, St. Louis, Missouri 63130 \\ ${ }^{5}$ SISSA, International School for Advanced Studies, Via Beirut 2-4, 34014 Trieste, Italy \\ ${ }^{6}$ INFN, Department of Physics, University of Trieste, Via A. Valerio 2, 34127 Trieste, Italy \\ ${ }^{7}$ National Center for Supercomputing Applications, Beckman Institute, 405 N. Mathews Avenue, Urbana, Illinois 61801 \\ ${ }^{8}$ Department of Physics, Aristotle University of Thessaloniki, Thessaloniki 54006, Greece \\ ${ }^{9}$ Physics Department, Chinese University of Hong Kong, Shatin, Hong Kong
}

(Received 9 October 2001; published 2 April 2002)

\begin{abstract}
This is the second in a series of papers on the construction and validation of a three-dimensional code for the solution of the coupled system of the Einstein equations and of the general relativistic hydrodynamic equations, and on the application of this code to problems in general relativistic astrophysics. In particular, we report on the accuracy of our code in the long-term dynamical evolution of relativistic stars and on some new physics results obtained in the process of code testing. The following aspects of our code have been validated: the generation of initial data representing perturbed general relativistic polytropic models (both rotating and nonrotating), the long-term evolution of relativistic stellar models, and the coupling of our evolution code to analysis modules providing, for instance, the detection of apparent horizons or the extraction of gravitational waveforms. The tests involve single nonrotating stars in stable equilibrium, nonrotating stars undergoing radial and quadrupolar oscillations, nonrotating stars on the unstable branch of the equilibrium configurations migrating to the stable branch, nonrotating stars undergoing gravitational collapse to a black hole, and rapidly rotating stars in stable equilibrium and undergoing quasiradial oscillations. We have carried out evolutions in full general relativity and compared the results to those obtained either with perturbation techniques, or with lower dimensional numerical codes, or in the Cowling approximation (in which all the perturbations of the spacetime are neglected). In all cases an excellent agreement has been found. The numerical evolutions have been carried out using different types of polytropic equations of state using either the rest-mass density only, or the rest-mass density and the internal energy as independent variables. New variants of the spacetime evolution and new high resolution shock capturing treatments based on Riemann solvers and slope limiters have been implemented and the results compared with those obtained from previous methods. In particular, we have found the "monotonized central differencing" limiter to be particularly effective in evolving the relativistic stellar models considered. Finally, we have obtained the first eigenfrequencies of rotating stars in full general relativity and rapid rotation. A long standing problem, such frequencies have not been obtained by other methods. Overall, and to the best of our knowledge, the results presented in this paper represent the most accurate long-term three-dimensional evolutions of relativistic stars available to date.
\end{abstract}

DOI: $10.1103 /$ PhysRevD.65.084024

PACS number(s): 04.30.Db, 04.25.Dm, 95.30.Sf

\section{INTRODUCTION}

Computational general relativistic astrophysics is an increasingly important field of research. Its development is being driven by a number of factors: firstly, the large amount of observational data by high-energy x-ray and $\gamma$-ray satellites such as Chandra, XMM and others [1]; secondly, the new generation of gravitational wave detectors coming online in the next few years [2]; and thirdly, the rapid increase in computing power through massively parallel supercomputers and the associated advance in software technologies, which make large-scale, multidimensional numerical simulations possible. Three-dimensional (3D) simulations of general relativistic astrophysical events such as stellar gravitational collapse or collisions of compact stars and black holes are needed to fully understand the incoming wealth of observa- tions from high-energy astronomy and gravitational wave astronomy. It is thus not surprising that in recent years hydrodynamical simulations of compact objects in numerical relativity have become the focus of several research groups [3-10].

In a previous paper [6] (paper I) we presented a 3D general-relativistic hydrodynamics code (GR_ASTRO) constructed for the NASA Neutron Star Grand Challenge Project [11]. The GR_ASTRO code has been developed by Washington University and the Albert Einstein Institute and has the capability of solving the coupled set of the Einstein equations and the general relativistic hydrodynamic (GR-Hydro) equations [12]. It has been built using the Cactus Computational Toolkit [13] constructed by the Albert Einstein Institute, Washington University and other institutes. Paper I presented our formulation for the GR-Hydro equations coupled either 
to the standard Arnowitt-Deser-Misner (ADM) [14] formulation of the Einstein equations or to a hyperbolic formulation of the equations [15]. It demonstrated the consistency and convergence of the code for a comprehensive sample of testbeds having analytic solutions. It gave a detailed analysis of twelve different combinations of spacetime and hydrodynamics evolution methods, including Roe's and other approximate Riemann solvers, as well as their relative performance and comparisons when applied to the various testbeds. The code as described and validated in paper I has been applied to various physical problems, such as those discussed in Refs. [7,16,17], and is now freely available [12].

The main purpose of this paper is to examine and validate our code in long-term, accurate simulations of the dynamics of isolated stars in strong gravitational fields. Single relativistic stars are indeed expected as the end-point of a number of astrophysical scenarios (such as gravitational collapse and binary neutron star merging) and should provide important information about strong field physics both through electromagnetic and gravitational wave emissions. A number of new numerical techniques have been incorporated in the present code leading to a much improved ability to simulate relativistic stars. These techniques concern both the evolution of the field equations, for which we have implemented new conformal-traceless formulations of the Einstein equations, and the evolution of the hydrodynamical variables, for which the use of the "monotonized central differencing" (MC) limiter has provided us with the small error growthrates necessary for simulations over several dynamical time scales.

More precisely, in this paper we focus on the accuracy of the code during long-term evolution of spherical and rapidly rotating stellar models. We also investigate the nonlinear dynamics of stellar models that are unstable to the fundamental radial mode of pulsation. Upon perturbation, the unstable models will either collapse to a black hole, or migrate to a configuration in the stable branch of equilibrium configurations (a behavior studied in the case of unstable boson stars [18]). In the case of collapse, we follow the evolution all the way down to the formation of a black hole, tracking the generation of its apparent horizon. In the case of migration to the stable branch, on the other hand, we are able to accurately follow the nonlinear oscillations that accompany this process and that can give rise to strong shocks. The ability to simulate large amplitude oscillations is important as we expect a neutron star formed in a supernova core-collapse $[19,20]$ or in the accretion-induced collapse of a white dwarf to oscillate violently in its early stages of life.

Particularly important for their astrophysical implications, we study the linear pulsations of spherical and rapidly rotating stars. The computed frequencies of radial, quasi-radial and quadrupolar oscillations are compared with the corresponding frequencies obtained with lower-dimensional numerical codes or with alternative techniques such as the Cowling approximation (in which the spacetime is held fixed and only the GR-Hyrdo equations are evolved) or relativistic perturbative methods. The comparison shows an excellent agreement confirming the ability of the code to extract physically relevant information from tiny perturbations. The suc- cessful determination of the eigenfrequencies for rapidly rotating stars computed with our code is noteworthy. Such frequencies have not been obtained before with the system being too complicated for perturbative techniques.

The simulations discussed here make use of two different polytropic equations of state (EOS). In addition to the standard "adiabatic" EOS, in which the pressure is expressed as a power law of the rest-mass density, we have carried out simulations implementing the "ideal fluid" EOS, in which the pressure is proportional to both the rest-mass density and the specific internal energy density. This latter choice increases the computational costs (there is one additional equation to be solved) but allows for the modeling of nonadiabatic processes, such as strong shocks and the conversion of bulk kinetic energy into internal energy, which are expected to accompany relativistic astrophysical events.

There are a number of reasons why we advocate the careful validation of general relativistic astrophysics codes. Firstly, the space of solutions of the coupled system of the Einstein and GR-Hydro equations is, to a large extent, unknown. Secondly, the numerical codes must solve a complicated set of coupled partial differential equations involving thousands of terms and there are plenty of chances for coding errors. Thirdly, the complex computational infrastructure needed for the use of the code in a massively parallel environment increases the risk of computational errors, a risk that can only be minimized through meticulous tests such as those presented here as well as in paper I. This paper, however, wants to be more than a list of testbeds: the results presented show that our current numerical methods are mature enough for obtaining answers to new and outstanding problems in the physics of relativistic stars.

The organization of this paper is as follows: the formulation of the differential equations for the spacetime and the hydrodynamics is briefly reviewed in Sec. II. Section III gives a short description of the numerical methods, with emphases on the new schemes introduced in this paper (in addition to those in paper I). Sections IV-VI represent the core of the paper and there we present the main results of our simulations. In Sec. IV, in particular, we focus our attention on the simulation of nonrotating relativistic stars. In Sec. V we consider the evolution of rotating stars. Section VI is dedicated to the extraction of gravitational waveforms generated by the non-radial pulsations of perturbed relativistic stars. In Sec. VII we summarize our results and conclusions. We use a spacelike signature $(-,+,+,+)$ and units in which $c=G=M_{\odot}=1$ (geometric units based on solar mass) unless explicitly specified. Greek indices are taken to run from 0 to 3 and Latin indices from 1 to 3 .

\section{BASIC EQUATIONS}

We give a brief overview of the system of equations in this section. We refer the reader to paper I for more details.

\section{A. Field equations}

In general relativity, the dynamics of the spacetime is described by the Einstein field equations $G_{\mu \nu}=8 \pi T_{\mu \nu}$, with 
$G_{\mu \nu}$ being the Einstein tensor and $T_{\mu \nu}$ the stress-energy tensor. Many different formulations of the equations have been proposed throughout the years, starting with the ADM formulation in 1962 [14]. In our code, we have implemented three different formulations of the field equations, including the ADM formulation, a hyperbolic formulation [15] and a more recent conformal-traceless formulation based on the ADM construction [21,22] (see also Ref. [23]).

In the ADM formulation [14], the spacetime is foliated with a set of non-intersecting spacelike hypersurfaces. Two kinematic variables relate the surfaces: the lapse function $\alpha$, which describes the rate of advance of time along a timelike unit vector $n^{\mu}$ normal to a surface, and the shift three-vector $\beta^{i}$ that relates the spatial coordinates of two surfaces. In this construction the line element reads

$$
d s^{2}=-\left(\alpha^{2}-\beta_{i} \beta^{i}\right) d t^{2}+2 \beta_{i} d x^{i} d t+\gamma_{i j} d x^{i} d x^{j} .
$$

The original ADM formulation casts the Einstein equations into a first-order (in time) quasi-linear [24] system of equations. The dependent variables are the 3-metric $\gamma_{i j}$ and the extrinsic curvature $K_{i j}$. The evolution equations read

$$
\begin{aligned}
\partial_{t} \gamma_{i j}= & -2 \alpha K_{i j}+\nabla_{i} \beta_{j}+\nabla_{j} \beta_{i}, \\
\partial_{t} K_{i j}= & -\nabla_{i} \nabla_{j} \alpha+\alpha\left[R_{i j}+K K_{i j}-2 K_{i m} K_{j}^{m}\right. \\
& \left.-8 \pi\left(S_{i j}-\frac{1}{2} \gamma_{i j} S\right)-4 \pi \rho_{\mathrm{ADM}} \gamma_{i j}\right] \\
& +\beta^{m} \nabla_{m} K_{i j}+K_{i m} \nabla_{j} \beta^{m}+K_{m j} \nabla_{i} \beta^{m},
\end{aligned}
$$

where $\nabla_{i}$ denotes the covariant derivative with respect to the 3-metric $\gamma_{i j}, R_{i j}$ is the Ricci curvature of the 3-metric, and $K \equiv \gamma^{i j} K_{i j}$ is the trace of the extrinsic curvature. In addition to the evolution equations, there are four constraint equations: the Hamiltonian constraint

$$
{ }^{(3)} R+K^{2}-K_{i j} K^{i j}-16 \pi \rho_{\mathrm{ADM}}=0,
$$

and the momentum constraints

$$
\nabla_{j} K^{i j}-\gamma^{i j} \nabla_{j} K-8 \pi j^{i}=0 .
$$

In Eqs. (2)-(5), $\rho_{\mathrm{ADM}}, j^{i}, S_{i j}, S \equiv \gamma^{i j} S_{i j}$ are the components of the stress-energy tensor projected onto the 3D hypersurface (for a more detailed discussion, see Ref. [25]).

As mentioned above, in addition to the two formulations described in paper I, we have recently implemented a conformal-traceless reformulation of the ADM system, as proposed by [21,22]. Details of our particular implementation of this formulation are extensively described in Ref. [23] and will not be repeated here. We only mention here that this formulation makes use of a conformal decomposition of the 3-metric, $\tilde{\gamma}_{i j}=e^{-4 \phi} \gamma_{i j}$ and the trace-free part of the extrinsic curvature, $A_{i j}=K_{i j}-\gamma_{i j} K / 3$, with the conformal factor $\phi$ chosen to satisfy $e^{4 \phi}=\gamma^{1 / 3} \equiv \operatorname{det}\left(\gamma_{i j}\right)^{1 / 3}$. In this formulation, as shown in Ref. [22], in addition to the evolution equations for the conformal three-metric $\tilde{\gamma}_{i j}$ and the conformal- traceless extrinsic curvature variables $\widetilde{A}_{i j}$, there are evolution equations for the conformal factor $\phi$, the trace of the extrinsic curvature $K$ and the "conformal connection functions" $\widetilde{\Gamma}^{i}$ (following the notation of Ref. [22]). We note that the final mixed, first and second-order, evolution system for $\left\{\phi, K, \tilde{\gamma}_{i j}, \widetilde{A}_{i j}, \widetilde{\Gamma}^{i}\right\}$ is not in any immediate sense hyperbolic [26]. In the original formulation of Ref. [21], the auxiliary variables $\widetilde{F}_{i}=-\Sigma_{j} \tilde{\gamma}_{i j, j}$ were used instead of the $\widetilde{\Gamma}^{i}$.

In Refs. [23,27] the improved properties of this conformal-traceless formulation of the Einstein equations were compared to the ADM system. In particular, in Ref. [23] a number of strongly gravitating systems were analyzed numerically with convergent high resolution shock capturing (HRSC) methods with total-variation-diminishing (TVD) schemes using the equations described in paper I. These included weak and strong gravitational waves, black holes, boson stars and relativistic stars. The results show that our treatment leads to a long-term numerical evolution of the many strongly gravitating systems. However, we have also found that the conformal-traceless formulation requires grid resolutions higher than the ones needed in the ADM formulation (with the driver technique [28]) to achieve the same accuracy. Because in long-term evolutions a small error growth-rate is the most desirable property, we have adopted the conformal-traceless formulation as our standard form for the evolution of the field equations.

\section{B. Hydrodynamic equations}

The GR-Hydro equations are obtained from the local conservation laws of the density current (continuity equation) and of the stress-energy tensor, which we assume to be that of a perfect fluid $T^{\mu \nu}=\rho h u^{\mu} u^{\nu}+P g^{\mu \nu}$, with $u^{\mu}$ being the fluid 4-velocity and $P$ and $h$ the (isotropic) pressure and the specific enthalpy, respectively. In our code the GR-Hydro equations are written as a first-order flux-conservative hyperbolic system $[29,6]$

$$
\partial_{t} \overrightarrow{\mathcal{U}}+\partial_{i} \vec{F}^{i}=\vec{S},
$$

where the evolved state vector $\overrightarrow{\mathcal{U}}$ is given, in terms of the primitive variables: the rest-mass density $\rho$, the 3-velocity $v^{i}=u^{i} / W+\beta^{i} / \alpha$ and the specific internal energy $\varepsilon$, as

$$
\overrightarrow{\mathcal{U}}=\left[\begin{array}{c}
\widetilde{D} \\
\widetilde{S}_{j} \\
\tilde{\tau}
\end{array}\right]=\left[\begin{array}{c}
\sqrt{\gamma} W \rho \\
\sqrt{\gamma} \rho h W^{2} v_{j} \\
\sqrt{\gamma}\left(\rho h W^{2}-P-W \rho\right)
\end{array}\right] .
$$

Here $\gamma$ is the determinant of the 3-metric $\gamma_{i j}$ and $W$ is the Lorentz factor, $W=\alpha u^{0}=\left(1-\gamma_{i j} v^{i} v^{j}\right)^{-1 / 2}$. Furthermore, the 3-flux vectors $\vec{F}^{i}$ are given by

$$
\vec{F}^{i}=\left[\begin{array}{c}
\alpha\left(v^{i}-\frac{1}{\alpha} \beta^{i}\right) \widetilde{D} \\
\alpha\left[\left(v^{i}-\frac{1}{\alpha} \beta^{i}\right) \widetilde{S}_{j}+\sqrt{\gamma} P \delta_{j}^{i}\right] \\
\alpha\left[\left(v^{i}-\frac{1}{\alpha} \beta^{i}\right) \tilde{\tau}+\sqrt{\gamma} v^{i} P\right]
\end{array}\right] .
$$


Finally, the source vector $\vec{S}$ is given by

$$
\vec{S}=\left[\begin{array}{c}
0 \\
\alpha \sqrt{\gamma} T^{\mu \nu} g_{\nu \sigma} \Gamma^{\sigma}{ }_{\mu j} \\
\alpha \sqrt{\gamma}\left(T^{\mu 0} \partial_{\mu} \alpha-\alpha T^{\mu \nu} \Gamma_{\mu \nu}^{0}\right)
\end{array}\right],
$$

where $\Gamma_{\mu \nu}^{\alpha}$ are the Christoffel symbols.

\section{Gauge conditions}

The code is designed to handle arbitrary shift and lapse conditions, which can be chosen as appropriate for a given spacetime simulation. More information about the possible families of spacetime slicings which have been tested and used with the present code can be found in Refs. $[6,23]$. Here, we limit ourselves to recall details about the specific foliations used in the present evolutions. In particular, we have used algebraic slicing conditions of the form

$$
\partial_{t} \alpha=-f(\alpha) \alpha^{2} K,
$$

with $f(\alpha)>0$ but otherwise arbitrary. This choice contains many well known slicing conditions. For example, setting $f$ $=1$ we recover the "harmonic" slicing condition, or by setting $f=q / \alpha$, with $q$ being an integer, we recover the generalized " $1+\log$ " slicing condition [30]. In particular, all of the simulations discussed in this paper are done using condition (10) with $f=2 / \alpha$ (we caution that "gauge pathologies" could develop with the " $1+\log$ " slicings, see Refs. $[31,32])$. The evolutions presented in this paper were carried out with the shift vector being either zero or constant in time.

\section{NUMERICAL METHODS}

We now briefly describe the numerical schemes used in our code. We will distinguish the schemes implemented in the evolution of the Einstein equations from those implemented in the evolution of the hydrodynamic equations. In both cases, the equations are finite-differenced on spacelike hypersurfaces covered with 3D numerical grids using Cartesian coordinates.

\section{A. Spacetime evolution}

As described in paper I, our code supports the use of several different numerical schemes [6,23]. Currently, a Leapfrog (non-staggered in time) and an iterative CrankNicholson scheme have been coupled to the hydrodynamic solver.

The Leapfrog method assumes that all variables exist on both the current time step $t^{n}$ and the previous time step $t^{n-1}$. Variables are updated from $t^{n-1}$ to $t^{n+1}$ (future time) evaluating all terms in the evolution equations on the current time step $t^{n}$. The iterative Crank-Nicholson solver, on the other hand, first evolves the data from the current time step $t^{n}$ to the future time step $t^{n+1}$ using a forward in time, centered in space first-order method. The solutions at steps $t^{n}$ and $t^{n+1}$ are then averaged to obtain the solution on the half time step $t^{n+1 / 2}$. This solution at the half time step $t^{n+1 / 2}$ is then used in a leapfrog step to re-update the solution at the final time step $t^{n+1}$. This process is then iterated. The error is defined as the difference between the current and previous solutions on the half time step $t^{n+1 / 2}$. This error is summed over all gridpoints and all evolved variables. Because the smallest number of iterations for which the iterative Crank-Nicholson evolution scheme is stable is three and further iterations do not improve the order of convergence [33,23], we do not iterate more than three times. Unless otherwise noted, all simulations reported in this paper use the iterative CrankNicholson scheme for the time evolution of the spacetime.

\section{B. Hydrodynamical evolution}

The numerical integration of the GR-Hydro equations is based on high-resolution shock-capturing (HRSC) schemes, specifically designed to solve nonlinear hyperbolic systems of conservation laws. These conservative schemes rely on the characteristic structure of the equations in order to build approximate Riemann solvers. In paper I we presented a spectral decomposition of the GR-Hydro equations, suitable for a general spacetime metric (see also Ref. [34]).

Approximate Riemann solvers compute, at every cellinterface of the numerical grid, the solution of local Riemann problems (i.e. the simplest initial value problem with discontinuous initial data). Hence HRSC schemes automatically guarantee that physical discontinuities developing in the solution (e.g., shock waves, which appear in core-collapse supernovae or in coalescing neutron star binaries) are treated consistently. HRSC schemes surpass traditional approaches $[3,8]$ which rely on the use of artificial viscosity to resolve such discontinuities, especially for large Lorentz factor flows. HRSC schemes have a high order of accuracy, typically second-order or more, except at shocks and extremal points. We refer the reader to $[35,36]$ for recent reviews on the use HRSC schemes in relativistic hydrodynamics.

One of the major advantages of using HRSC schemes is that we can take advantage of the many different algorithms that have been developed and tested in Newtonian hydrodynamics. In this spirit, our code allows for three alternative ways of performing the numerical integration of the hydrodynamic equations: (i) using a flux-split method [37]; (ii) using Roe's approximate Riemann solver [38], and (iii) using Marquina's flux-formula [39]. The different methods differ simply in the way the numerical fluxes at the cell-interfaces are calculated in the corresponding flux-formula. The code uses slope-limiter methods to construct second-order total variation diminishing (TVD) schemes [40] by means of monotonic piecewise linear reconstructions of the cellcentered quantities to the left $(\mathrm{L})$ and right $(\mathrm{R})$ sides of every cell-interface for the computation of the numerical fluxes. More precisely, $\overrightarrow{\mathcal{U}}_{i}^{R}$ and $\overrightarrow{\mathcal{U}}_{i+1}^{L}$ are computed to second-order accuracy as follows:

$$
\begin{aligned}
\overrightarrow{\mathcal{U}}_{i}^{R} & =\overrightarrow{\mathcal{U}}_{i}+\sigma_{i}\left(x_{i+(1 / 2)}-x_{i}\right) \\
\overrightarrow{\mathcal{U}}_{i+1}^{L} & =\overrightarrow{\mathcal{U}}_{i+1}+\sigma_{i+1}\left(x_{i+(1 / 2)}-x_{i+1}\right)
\end{aligned}
$$

where $x$ denotes a generic spatial coordinate. We have fo- 
cused our attention on two different types of slope limiters, the standard "minmod" limiter and the "monotonized central-difference" (MC) limiter [41]. In the first case, the slope $\sigma_{i}$ is computed according to

$$
\sigma_{i}=\operatorname{minmod}\left(\frac{\overrightarrow{\mathcal{U}}_{i}-\overrightarrow{\mathcal{U}}_{i-1}}{\Delta x}, \frac{\overrightarrow{\mathcal{U}}_{i+1}-\overrightarrow{\mathcal{U}}_{i}}{\Delta x}\right),
$$

where $\Delta x$ denotes the cell spacing. The minmod function of two arguments is defined by

$$
\operatorname{minmod}(a, b) \equiv \begin{cases}a & \text { if }|a|<|b| \text { and } a b>0, \\ b & \text { if }|b|<|a| \text { and } a b>0, \\ 0 & \text { if } a b \leqslant 0 .\end{cases}
$$

On the other hand, the MC slope limiter (which was not included in the previous version of the code discussed in paper I) does not reduce the slope as severely as minmod near a discontinuity and, therefore, a sharper resolution can be obtained. In this case the slope is computed as

$$
\sigma_{i}=\operatorname{MC}\left(\frac{\overrightarrow{\mathcal{U}}_{i}-\overrightarrow{\mathcal{U}}_{i-1}}{\Delta x}, \frac{\overrightarrow{\mathcal{U}}_{i+1}-\overrightarrow{\mathcal{U}}_{i}}{\Delta x}\right),
$$

where the MC function of two arguments is defined by

$$
\begin{aligned}
& \operatorname{MC}(a, b)
\end{aligned}
$$

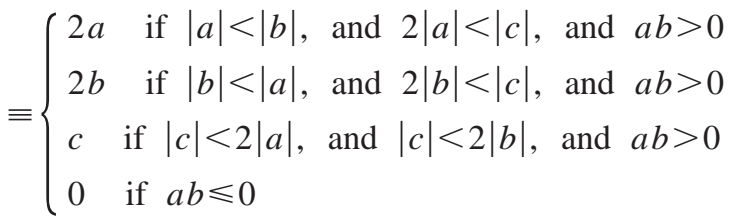

and where $c \equiv(a+b) / 2$. Both schemes provide the desired second-order accuracy for smooth solutions, while still satisfying the TVD property. In Sec. IV A we will report on a comparison between the two algorithms and justify the use of the MC slope limiter as our preferred one.

\section{Equations of state}

As mentioned in the Introduction, to explore the behavior of our code in long-term evolutions of equilibrium configurations, we used two different polytropic equations of state and at various central rest-mass densities. In particular, we have implemented both an adiabatic (or zero temperature) EOS

$$
P=K \rho^{\Gamma}=K \rho^{1+1 / N},
$$

and as a so-called "ideal fluid" EOS

$$
P=(\Gamma-1) \rho \varepsilon,
$$

where $K$ is the polytropic constant, $\Gamma$ the polytropic index and $N \equiv(\Gamma-1)^{-1}$ the polytropic exponent. The ideal fluid EOS (16) depends on both the rest-mass density $\rho$ and on the specific internal energy $\varepsilon$; it corresponds to allowing the polytropic coefficient $K$ in adiabatic EOS (15) to be a func- tion of entropy. The use of an adiabatic EOS with a constant $K$ is computationally less expensive and is physically reasonable when modeling configurations that are in near equilibrium, such as stable stellar models in quasi-equilibrium evolutions. There are however dynamical processes, such as those involving nonlinear oscillations and shocks, in which the variations in the energy entropy cannot be neglected. The simulations discussed in Sec. IV C, where both equations of state (15),(16) are used for the same configuration, give direct evidence of how a more realistic treatment of the internal energy of the system can produce qualitatively different results.

The increased accuracy in the physical description of the dynamical system comes with a non-negligible additional computational cost. It involves the solution of an additional equation (i.e. the evolution equation for the specific internal energy $\varepsilon$ ), increasing the total number of GR-Hydro equations from four to five and making accurate long-term evolutions considerably harder.

\section{Boundary conditions}

In our general-purpose code, a number of different boundary conditions can be imposed for either the spacetime variables or for the hydrodynamical variables. We refer the reader to $[6,23]$ for details. In all of the runs presented in this paper we have used static boundary conditions for the hydrodynamical variables and radiative outgoing boundary conditions for the spacetime variables. The only exception to this is the evolution of rotating stars (see Sec. V), for which the spacetime variables have also been held fixed at the outer boundary.

\section{SPHERICAL RELATIVISTIC STARS}

We turn next to the description of the numerical evolutions of relativistic star configurations. We start by considering spherical models.

\section{A. Long-term evolution of stable configurations}

Using isotropic coordinates $(t, r, \theta, \phi)$, the metric describing a static, spherically symmetric relativistic star reads

$$
d s^{2}=-e^{2 \nu} d t^{2}+e^{2 \lambda}\left(d r^{2}+r^{2} d \theta^{2}+r^{2} \sin ^{2} \theta d \phi^{2}\right),
$$

where $\nu$ and $\lambda$ are functions of the radial coordinate $r$ only. The form of the metric component $g_{r r}$ is much simpler in these coordinates than in Schwarzschild coordinates, which are often used to describe a Tolman-Oppenheimer-Volkoff (TOV) equilibrium stellar solution. In addition, $g_{r r}$ is not constrained to be equal to unity at the center of the stellar configuration, as in Schwarzschild coordinates. We have found that these two properties of the isotropic coordinates are very beneficial to achieve long-term numerical evolutions of relativistic stars. Therefore, all simulations of spherical relativistic stars shown in this paper have been performed adopting the line element (17) expressed in Cartesian coordinates. 


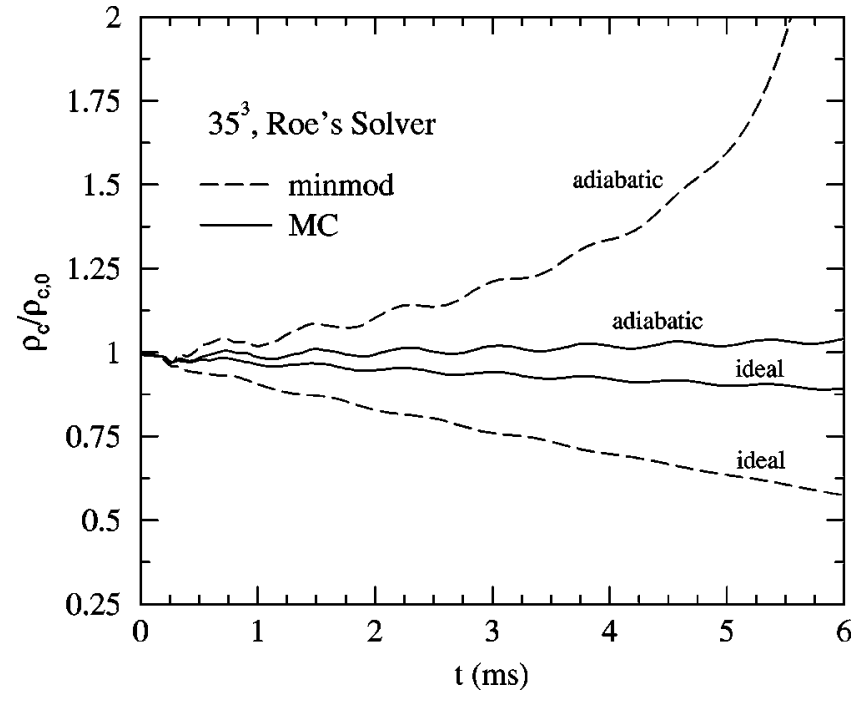

FIG. 1. Evolution of the central rest-mass density $\rho_{c}$ (in units of the initial central rest-mass density $\rho_{c, 0}$ ) for a nonrotating star with gravitational mass $M=1.65 M_{\odot}$. Using Roe's approximate Riemann solver, the figure shows a comparison in the use of the minmod and of the MC slope limiters for both the ideal fluid and the adiabatic EOS.

Although the initial configurations refer to stellar models in stable equilibrium, the truncation errors at the center and at the surface of the star excite small radial pulsations that are damped in time by the numerical viscosity of the code. Moreover, these pulsations are accompanied by a secular evolution of the values of the central rest-mass density away from its initial value. Similar features have been reported in Refs. [42,43]. These features converge away at the correct rate with increasing grid resolution and do not influence the long-term evolutions. Moreover, the secular evolution of the central rest-mass density varies according to the EOS adopted: when using the ideal fluid EOS, we have observed that the secular drift of the central rest-mass density is towards lower densities. However, if we enforced the adiabatic condition (which is justified for the case of a nearequilibrium evolution), we have observed that the dominant truncation error has opposite sign and the central rest-mass density evolves towards larger values. The different behavior shown by the two equations of state should not be surprising. The stellar models we are solving for are in fact static only in the ideal limit of infinite numerical resolution. On a finite grid, however, these models are not static and indeed show pulsations in their fundamental mode of oscillation (see the subsequent discussion). In this case it is natural to expect that different equations of state could lead to qualitatively different secular behaviors.

This is shown in Fig. 1 where we plot the evolution of a TOV star with gravitational mass $M=1.65 M_{\odot}$, constructed with an $N=1$ polytrope. In our units, the polytropic constant is $K=123.5$ and the central rest-mass density of the star is $\rho_{c}=1.00 \times 10^{-3}$. For these tests, a very coarse grid of $35^{3}$ gridpoints in octant symmetry is sufficient and allows the major effects to be revealed with minimal computational costs. The outer boundary is placed at about $1.7 r_{s}$ (where $r_{s}$

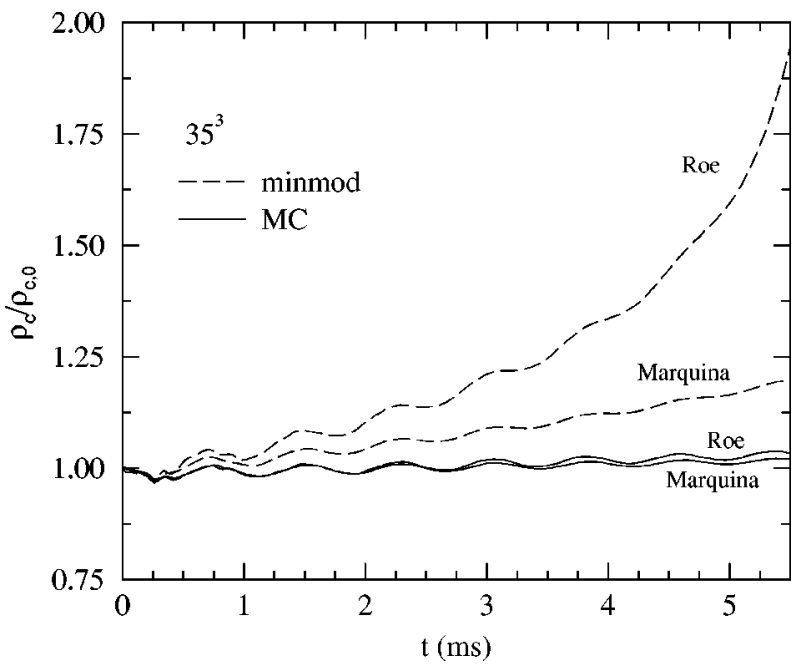

FIG. 2. Evolution of the normalized central rest-mass density $\rho_{c}$ for a nonrotating $M=1.65 M_{\odot}$ star. Different lines show a comparison between Roe's Riemann solver and Marquina's flux formula for different slope limiters.

is the isotropic coordinate radius of the star). We use radiative boundary conditions with a $1 / r$ fall-off. Irrespective of the slope limiter used, the magnitude of the secular drift observed in the central rest-mass density evolution is roughly a factor of two smaller for the adiabatic EOS than for the ideal fluid EOS. As a result, in all of the evolutions of stable configurations which remain close to equilibrium (such as pulsating stars, with no shock developing), the adiabatic EOS is preferred.

Figure 1 also gives a comparison of the use of the minmod and the MC slope limiters in the evolution of the normalized central rest-mass density. For both the ideal fluid and the adiabatic EOS, the MC limiter shows a significantly smaller secular increase in the central rest-mass density, as compared to the minmod one. The simulations in Fig. 1 employed Roe's approximate Riemann solver in the fluid evolution scheme and this is then compared to Marquina's fluxformula in Fig. 2 for the evolution of the central rest-mass density. The secular increase is significantly smaller when using Marquina's flux-formula than when using Roe's solver, and this is especially noticeable for the minmod slope limiter. A comparison of the increase of the maximum error in the Hamiltonian constraint after several ms of evolution (not shown here) indicates that it is about $80 \%$ smaller with Marquina than with Roe, when using the adiabatic EOS. As a result of the above comparisons, we have adopted Marquina's scheme with the MC slope limiter as our preferred scheme for evolution of the GR-Hydro equations. Unless otherwise noted, all of the simulations presented in this paper have been obtained with such a scheme.

Next, we show in Fig. 3 the long-term evolution of the central rest-mass density for three different grid resolutions. For this, we consider a nonrotating $N=1$ polytropic star with gravitational mass $M=1.4 M_{\odot}$, circumferential radius $R$ $=14.15 \mathrm{~km}$, central rest-mass density $\rho_{c}=1.28 \times 10^{-3}$ and $K=100$. The different simulations used $32^{3}, 64^{3}$ and $96^{3}$ gridpoints with octant symmetry and with the outer boundary 


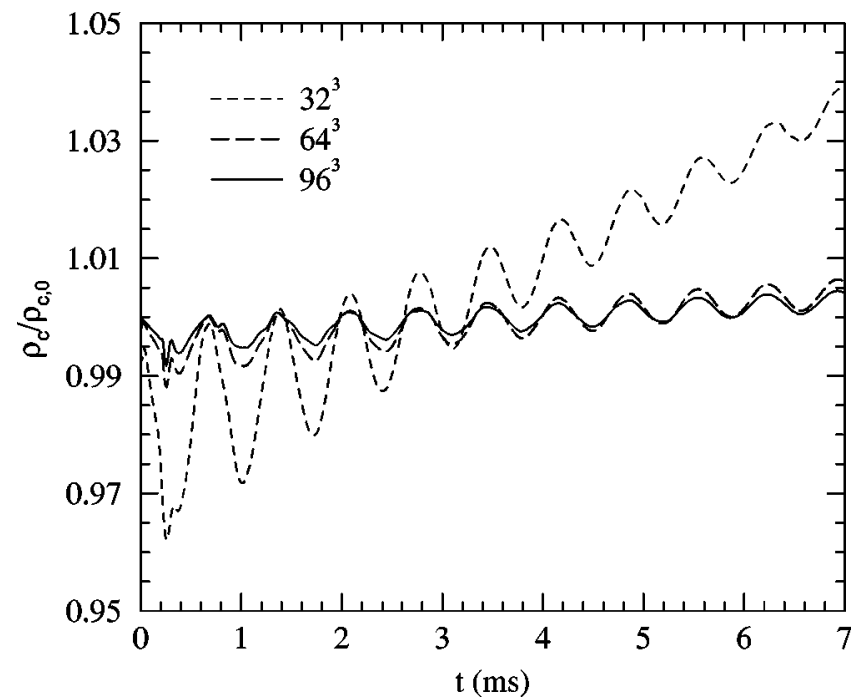

FIG. 3. Time evolution of the normalized central rest-mass density at three different grid resolutions $\left(32^{3}, 64^{3}\right.$ and $96^{3}$ gridpoints, respectively), for an $M=1.4 M_{\odot}, N=1$ relativistic, spherical polytrope. The evolution of the central rest-mass density is mainly modulated by the fundamental radial mode of oscillation of the star. The initial amplitude of the oscillation converges to zero at secondorder, while the secular increase in the central rest-mass density converges away at almost second-order.

placed at $1.7 r_{s}$. These grid resolutions correspond to about 19, 38 and 56 gridpoints per star radius, respectively. Figure 3 shows the oscillations in the central rest-mass density and the secular evolution away from the initial value mentioned above. The oscillations are produced by the first-order truncation error at the center and the surface of the star (our hydrodynamical evolution schemes are globally second order, but only first-order at local extrema; see related discussions in Ref. [23], where long-term convergence tests are presented) but both the amplitude of the initial oscillation and the rate of the secular change converge to zero at nearly second-order with increasing grid resolution.

Note that the evolutions shown in Figs. 3-5 extend to 7 $\mathrm{ms}$, corresponding to about 10 dynamical times (taking the fundamental radial mode period of pulsation as a measure of the dynamical time scale), significantly longer than, say, the ones reported by other authors $[8,44]$. Our evolutions are limited by the time available (a simulation with $96^{3}$ gridpoints and up to $7 \mathrm{~ms}$ takes about 40 hours on a 128 processor Cray-T3E supercomputer). We have found that for a resolution of $96^{3}$, the final central density, averaged over the last three periods of oscillations, is just $0.25 \%$ larger than the initial central density.

For the same configuration, we show, in Fig. 4, the time evolution of the L2-norm of the violation of the Hamiltonian constraint at the three different grid resolutions. Also in this case, the violation of the Hamiltonian constraint converges to zero at nearly second-order with increasing grid resolution.

In Fig. 5, we show other aspects of the accuracy of the simulation with $96^{3}$ gridpoints, by comparing the initial profiles of the rest-mass density $\rho$ and of the lapse function $\alpha$ of the TOV star with those obtained after $7 \mathrm{~ms}$ of evolution.

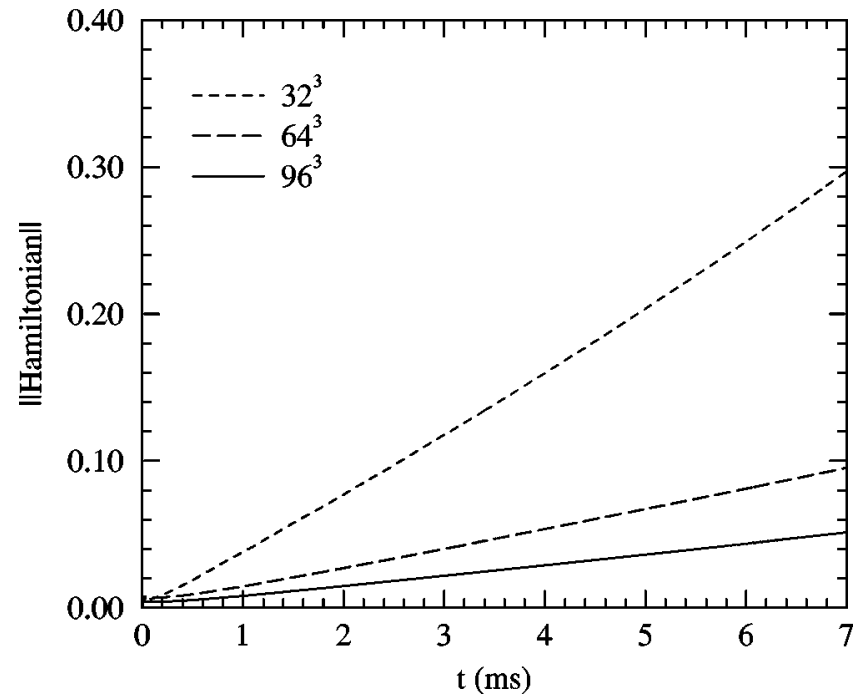

FIG. 4. Convergence of the L2-norm of the Hamiltonian constraint, at three different grid resolutions $\left(32^{3}, 64^{3}\right.$ and $96^{3}$ gridpoints, respectively), for an $M=1.4 M_{\odot}, N=1$ polytropic spherical relativistic star. The rate of convergence is close to second-order with increasing grid resolution.

The small deviations from the original profiles are worth emphasizing. The small inset shows a magnification of the rapid change in the gradient of the rest-mass density profile at the surface of the star.

\section{B. Radial pulsations}

As mentioned in the previous section, the truncation errors of the hydrodynamical schemes used in our code trigger radial pulsations of the initially static relativistic star (see Ref. [45] for a review). These pulsations are initiated at the

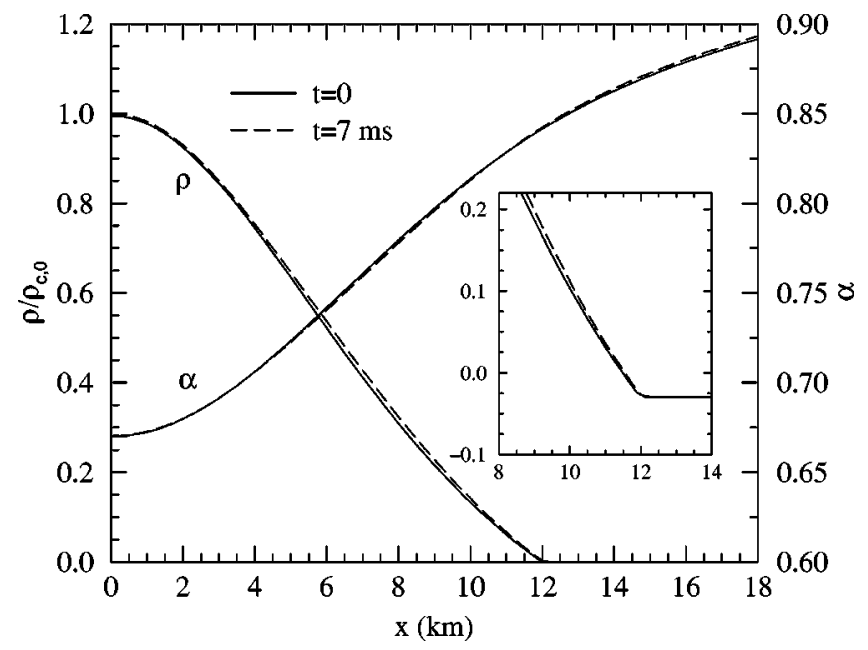

FIG. 5. Variation of the original profiles along the $x$-axis of the rest-mass density (left vertical axis) and lapse function (right vertical axis), for an $M=1.4 M_{\odot}, N=1$ polytropic spherical relativistic star, after $7 \mathrm{~ms}$ of evolution. A $96^{3}$ grid in octant symmetry was used in the simulation. The small inset shows a magnification of the rapid change in the gradient of the rest-mass density profile at the surface of the star. 


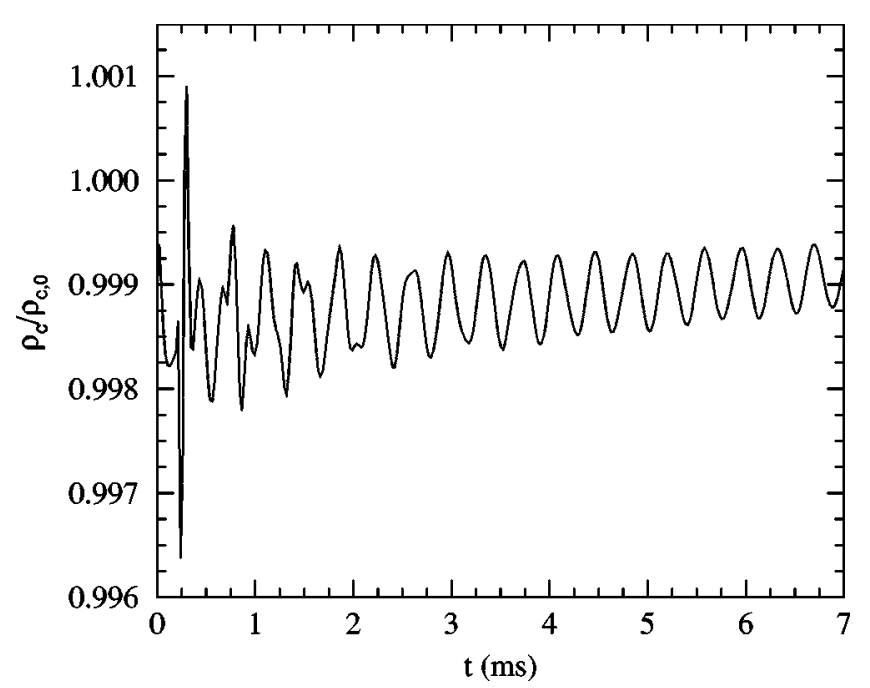

FIG. 6. Time evolution of the central rest-mass density of an $M=1.4 M_{\odot}, N=1$ polytropic spherical relativistic star. In this simulation the spacetime is held fixed and the hydrodynamic variables have been evolved on a numerical grid of $96^{3}$ gridpoints. The evolution is a superposition of radial normal modes of pulsation, excited by truncation errors of the hydrodynamical scheme. Higher overtones are damped faster by the small but non-zero numerical viscosity.

surface of the star, where the gradients of the rest-mass density are the largest (cf. Fig. 5). Because gravitational waves cannot be emitted through the excitation of radial pulsations of nonrotating relativistic stars, these pulsations are damped only by the numerical viscosity of the code in numerical simulations of inviscid stars. In treatments more dissipative than the HRSC schemes used in our code, such as those using artificial viscosity or particle methods (e.g. smoothed particle hydrodynamics), these oscillations will be damped significantly faster.

In order to test the properties of the long-term hydrodynamical evolution separately from those of the spacetime evolution, we have first examined the small-amplitude radial pulsations in a fixed spacetime of an initially static relativistic star. As initial data, we use the $M=1.4 M_{\odot}$ polytropic star of the previous section. We show, in Fig. 6, the evolution up to $7 \mathrm{~ms}$ of the normalized star's central rest-mass density with a numerical grid of $96^{3}$ gridpoints. The amplitude of the excited pulsations in this purely hydrodynamical evolution is minute (less than 1 part in 200) and is significantly smaller than the corresponding amplitude in a coupled hydrodynamical and spacetime evolution (compare the vertical axes of Figs. 3 and 6).

A closer look at Fig. 6 reveals that the evolution of the central rest-mass density is a superposition of different radial normal modes of pulsation. The higher-frequency modes are damped faster, so that after a certain time the evolution proceeds mainly in the fundamental mode of pulsation. Note also the small damping rate of the fundamental pulsation mode, indicating the small effective numerical viscosity of our HRSC hydrodynamical scheme. The evolution towards larger values of the central rest-mass density is similar to that discussed in Sec. IV A but less pronounced in this case. At a resolution of $96^{3}$ gridpoints, the secular change in the average central rest-mass density is less than $0.02 \%$ for the total evolution time shown.

The use of truncation error as an initial perturbation deserves commenting on. The oscillations caused by truncation error will converge away with increasing resolution, hence the overall oscillation amplitude can carry no physical information about the system. However, the frequencies and normalized eigenfuntions of particular normal-modes of oscillation of the star are physical (in the sense that they match the eigenfrequencies and eigenfunctions calculated through perturbative analyses) and can be extracted from these simulations by carrying out a Fourier transform of the time evolution of the radial velocity or of the rest-mass density. As the small-amplitude pulsations are in the linear regime, the eigenfunctions can be normalized arbitrarily (e.g. to 1.0 at the surface of the star). At increasing resolution, the solution converges to the mode-frequencies and to the normalized eigenfunctions, even though the overall oscillation amplitude converges to zero. Such evolutions are useful for extracting the properties of linear normal-modes of oscillation, as long as the resolution is fine enough that the pulsations excited by truncation errors are in the linear regime and as long as the resolution is coarse enough that the various local first and second order truncation errors of the numerical scheme result in a time evolution that is dominated by a sum of normal modes (at very fine resolutions the Fourier transform of the time evolution would be very small and thus have a very noisy power spectrum due to roundoff errors, in which case the physical normal-mode frequencies would be difficult to extract - this has not been the case for the resolutions used in this paper). We also note that different variants of our hydrodynamical evolution schemes excite the various physical normal-modes at different amplitudes. For example, second order schemes employing the minmod limiter tend to clearly excite a large number of high-frequency overtones, whereas the use of the MC limiter results in the clear excitation of only a few low-frequency overtones and a more noisy power spectrum at higher frequencies (for the resolutions used in this paper). This difference in behavior is due to the differences in the local truncation errors inherent in these numerical schemes.

The radial pulsations are a sum of eigenmodes of pulsation. Since the radial pulsations triggered by truncation errors have a small amplitude, one can compare the frequencies with that computed by linear perturbation theory [43] or with hydrodynamical evolutions of similar models in 2D [42,43]. In this way we can validate that the "artificial" perturbations produced by the truncation errors do excite "physical" modes of oscillation for a relativistic star. However, before discussing the results of this comparison, it is important to emphasize that the identification of the frequency peaks in the Fourier transform of the time evolution of a given variable with physical frequencies must be done with care. A real pulsation frequency must be global (the same at every point in the star, at least for discrete normal mode frequencies) and it should appear in the time evolution of different physical 


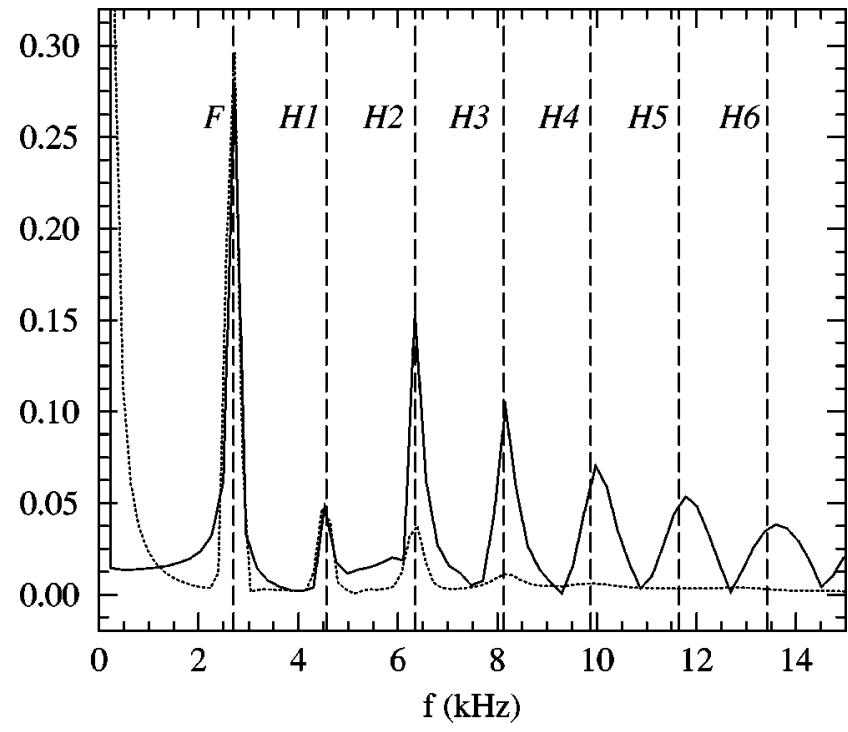

FIG. 7. Fourier transform of the central rest-mass density evolution of an $M=1.4 M_{\odot}, N=1$ polytropic spherical relativistic star, in a fixed spacetime evolution. Here $F$ represents the fundamental normal mode frequency, while $H 1-H 6$ indicate the first six overtones. The frequency peaks in the power spectrum are in excellent agreement with the radial normal mode frequencies (shown here as dashed vertical lines) computed with an independent 2D code using spherical polar coordinates. The solid and dotted lines were computed with $96^{3}$ and $64^{3}$ gridpoints, respectively. The units of the vertical axis are arbitrary.

quantities describing the star's structure and dynamics. To eliminate possible ambiguities, we have carried out our frequency identification procedure for different variables and at different positions in the star.

Figure 7 shows the Fourier transform of the time evolution of the central rest-mass density of the same initial model as in Fig. 6, but using the minmod limiter (which gives a clearer excitation of the higher overtones). We indicate with $F$ the fundamental normal mode frequency and with $H 1-H 6$ the next six higher frequency modes (overtones). We have also compared the frequency peaks in the Fourier spectrum to both the normal mode frequencies expected by linear perturbation theory in the Cowling approximation (see Ref. [46]) and to the frequencies computed with an independent 2D axisymmetric nonlinear code [43], which uses the same HRSC schemes but in spherical polar coordinates (shown as dashed vertical lines in Fig. 7).

As can be seen from Table I, the agreement is extremely good. The relative difference between the $3 \mathrm{D}$ and $2 \mathrm{D}$ results at this grid resolution is better than $1 \%$ up to $(H 4)$ and slightly larger for higher frequencies ( $\mathrm{H} 5$ and $\mathrm{H6}$ ), which become under-resolved at this grid resolution. This excellent agreement is a significant test for the correct implementation of the hydrodynamical evolution schemes in our code, and is an indication of the level of accuracy we can achieve, resolving and following these small deviations away from the equilibrium configuration. As one would expect, lower or higher resolution runs (e.g. with $64^{3}$ or $144^{3}$ gridpoints), which have intrinsically larger or smaller perturbation amplitudes, respectively, reproduce the peaks in the power spectrum
TABLE I. Comparison of small-amplitude radial pulsation frequencies obtained with the present 3D nonlinear evolution code with frequencies obtained with an independent $2 \mathrm{D}$ code. Both codes evolve the GR-Hydro equations in a fixed spacetime and for an equilibrium model of an $N=1$ relativistic polytrope with $M / R$ $=0.15$.

\begin{tabular}{cccc}
\hline \hline Mode & $\begin{array}{c}\text { Present 3D code } \\
(\mathrm{kHz})\end{array}$ & $\begin{array}{c}2 \mathrm{D} \text { code } \\
(\mathrm{kHz})\end{array}$ & $\begin{array}{c}\text { Relative Difference } \\
(\%)\end{array}$ \\
\hline$F$ & 2.696 & 2.701 & 0.2 \\
$H 1$ & 4.534 & 4.563 & 0.6 \\
$H 2$ & 6.346 & 6.352 & 0.1 \\
$H 3$ & 8.161 & 8.129 & 0.4 \\
$H 4$ & 9.971 & 9.875 & 1.0 \\
$H 5$ & 11.806 & 11.657 & 1.3 \\
$H 6$ & 13.605 & 13.421 & 1.7 \\
\hline \hline
\end{tabular}

shown in Fig. 7 (see dotted line in Fig. 7), which corresponds to an evolution with $64^{3}$ grid-points.

After establishing the accuracy of the long-term evolution of the GR-Hydro equations, we have examined the eigenfrequencies of the radial pulsations of spherical stars in coupled hydrodynamical and spacetime evolutions. A Fourier transform of the evolution of the radial velocity (for the same equilibrium model as the one discussed before) is shown in Fig. 8. Again in this case, we have been able to identify several frequency peaks in the Fourier spectrum with the normal mode frequencies obtained with linear perturbation techniques [47]. A detailed comparison of these frequencies

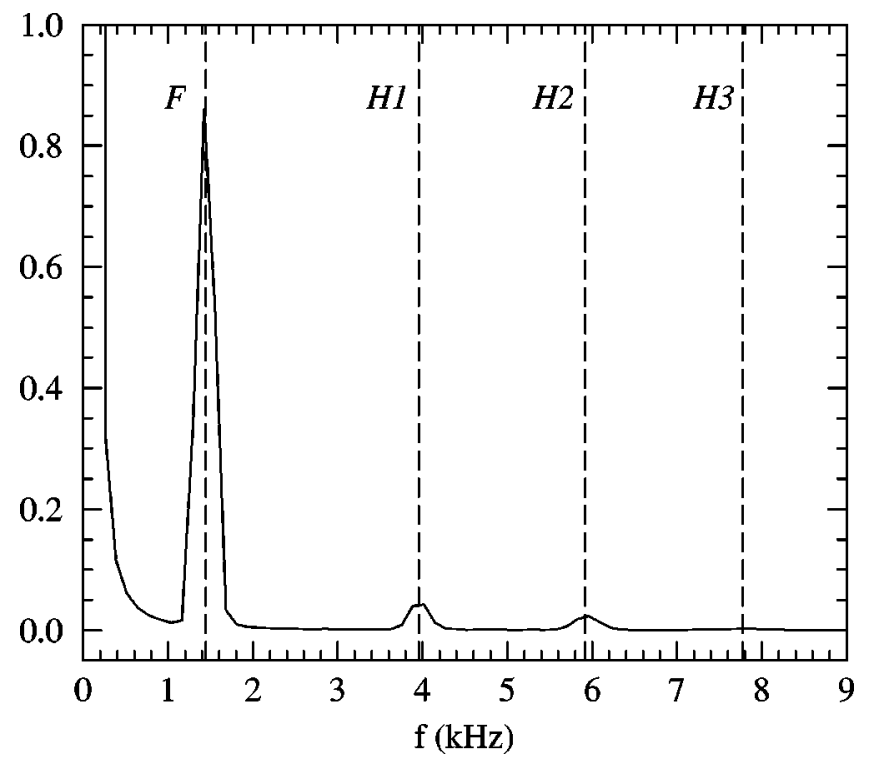

FIG. 8. Fourier transform of the evolution of the radial velocity for an $M=1.4 M_{\odot}, N=1$ polytropic spherical relativistic star in a coupled spacetime and hydrodynamical evolution. The frequency peaks in the spectrum are in excellent agreement with the radial normal mode frequencies computed by perturbation theory (shown here as dashed vertical lines). As in Fig. 7, here $F$ represents the fundamental normal mode frequency, while $H 1-H 3$ are the next three higher frequency modes. The units of the vertical axis are arbitrary. 
TABLE II. Comparison of small-amplitude radial pulsation frequencies obtained with the present 3D nonlinear evolution code with linear perturbation mode frequencies, in fully coupled evolutions. The equilibrium model is a nonrotating $N=1$ relativistic polytrope with $M / R=0.15$.

\begin{tabular}{cccc}
\hline \hline Mode & $\begin{array}{c}\text { Present 3D code } \\
(\mathrm{kHz})\end{array}$ & $\begin{array}{c}\text { Perturbation code } \\
(\mathrm{kHz})\end{array}$ & $\begin{array}{c}\text { Relative Difference } \\
(\%)\end{array}$ \\
\hline$F$ & 1.450 & 1.442 & 0.6 \\
$H 1$ & 3.958 & 3.955 & 0.0 \\
$H 2$ & 5.935 & 5.916 & 0.3 \\
$H 3$ & 7.812 & 7.776 & 0.4 \\
\hline \hline
\end{tabular}

is shown in Table II. The agreement is again excellent. Note the rather large differences between the frequencies shown in Tables I and II. The Cowling approximation is rather inaccurate for the lowest radial mode-frequencies [48], but is increasingly more accurate for nonradial pulsations or for higher frequencies [48].

All of the results discussed so far refer to simulations involving stable relativistic configurations. In the following section we consider numerical evolutions of relativistic stars which are initially in an unstable equilibrium.

\section{Migration of unstable configurations to the stable branch}

The numerical evolution of a nonrotating, relativistic star in an equilibrium unstable to the fundamental radial mode of pulsation is mainly determined by the numerical truncation errors that cause it to evolve away from its initial configuration. Depending on the type of perturbation, the star can either collapse to a black hole or expand and migrate to the stable branch of the sequence of equilibrium models, reaching a new, stable equilibrium configuration with approximately the same rest-mass of the perturbed star. We have therefore constructed a model of an $N=1, K=100$ polytropic star with rest-mass $M_{0}=1.535 M_{\odot}\left(M=1.447 M_{\odot}\right)$ and a central rest-mass density $\rho_{c}=8.0 \times 10^{-3}$, which is larger than the central rest-mass density of the maximummass stable model. The star is therefore initially in an unstable equilibrium (see the inset of Fig. 9) and under the perturbation introduced by the truncation error, it expands, evolving rapidly to smaller central rest-mass densities, until it reaches the stable branch of equilibrium configurations. An analogous behavior has been observed in numerical simulations of relativistic boson stars [18] (see also Ref. [49] for recent numerical simulations of expanding unstable boson stars).

In a realistic astrophysical scenario, a stable neutron star can accrete matter e.g. from a companion star in a binary system or from infalling matter after its formation in a supernova core-collapse. The star would then secularly move towards larger central densities along the stable branch of equilibrium configurations, exceed the maximum-mass limit and collapse to a black hole. No secular mechanism could evolve the star to the unstable branch. In this respect, the migration mechanism discussed here cannot occur in practice. Nevertheless, it provides a consistent solution of the

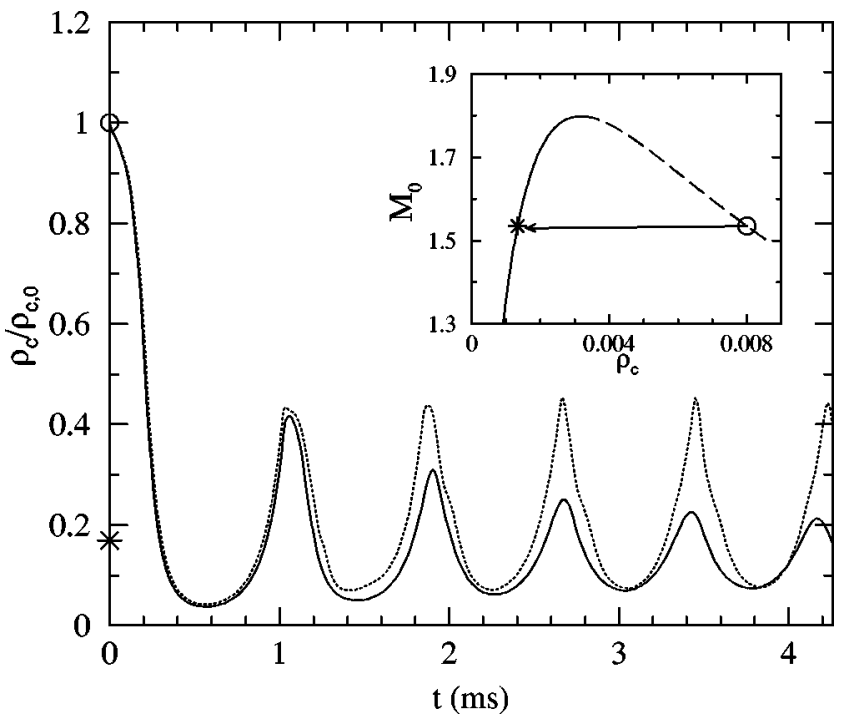

FIG. 9. Evolution of the (normalized) central rest-mass density $\rho_{c}$ during the migration of an unstable relativistic star to a stable model with the same rest-mass. When an adiabatic EOS is used (dotted line) the difference in gravitational binding energy between the unstable and stable models is periodically converted in bulk kinetic energy through highly nonlinear, nearly constant amplitude pulsations. In contrast, when an ideal fluid EOS is used (solid line), the gravitational binding energy is gradually converted into internal energy via shock heating. As a result, the oscillations are damped and the heated stable equilibrium model approaches a central density slightly smaller than the rest-mass density of a zero temperature star of the same rest-mass (indicated by an asterisk on the left vertical axis).

initial value problem and represents an important test of the accuracy of the code in a highly dynamical and non-adiabatic evolution. We use such an initial data set to study large amplitude oscillations of relativistic stars, which cannot be treated accurately by linear perturbation theory. Large amplitude oscillations about a configuration on the stable branch could occur after a supernova core-collapse [20] or after an accretion-induced collapse of a white dwarf. While the actual set of quasi-normal modes excited will depend on the excitation process, the ability to simulate large amplitude oscillations is important.

Figure 9 shows the evolution of the central rest-mass density $\rho_{c}$ normalized to its initial value and up to a final time of $4.26 \mathrm{~ms}$. On a very short dynamical time scale of $0.5 \mathrm{~ms}$ the star has expanded and has its central density dropped to about $3 \%$ of its initial central rest-mass density. Note that this is less than the central rest-mass density, $\rho_{c}=1.35$ $\times 10^{-3}$, of the stable model of the same rest-mass, which is indicated with an asterisk on the vertical axis of Fig. 9. During the rapid decrease of the central rest-mass density, the star acquires a large radial momentum. The star then enters a phase of large amplitude radial oscillations about the stable equilibrium model with the same rest-mass. Because the unstable and stable models have rather different degrees of compactness, the migration to the stable branch will be accompanied by the release of a significant amount of gravitational binding energy which could either be converted to 


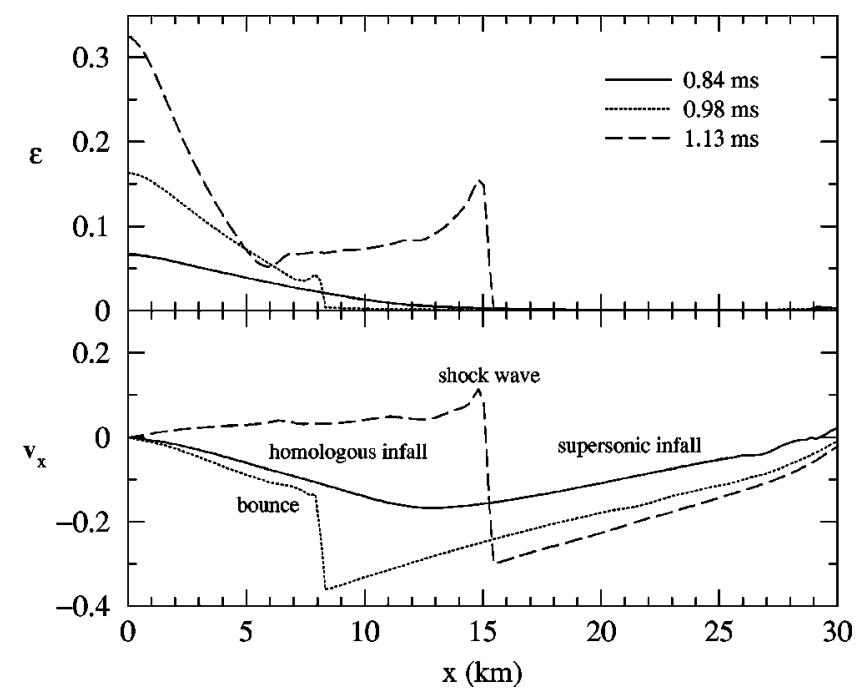

FIG. 10. Shock formation in the outer core mantle, during the first bounce at equilibrium densities of an unstable star, evolved with an ideal fluid EOS. The top and bottom panels show the internal energy $\epsilon$ and radial velocity $v_{x}$, respectively, at three different times: the homologous infall phase, the inner core bounce and the outwards shock propagation. The oscillations of the inner core are damped by shock heating.

bulk kinetic energy or to internal energy depending on the choice of EOS.

In order to investigate both responses, we have performed two different evolutions of the same initial model. In the first case (the "adiabatic EOS" in Fig. 9), we have enforced the adiabatic condition during the evolution, i.e. we have assumed that the star remains at zero temperature following an adiabatic EOS. As shown in Fig. 9 with a dotted line, in this case the star behaves like a compressed spring which is allowed to expand, oscillating with a nearly constant amplitude. This indicates that the star periodically converts all of the excess gravitational binding energy into the kinetic energy and vice versa. As the oscillations are highly nonlinear, the restoring force is weaker at higher densities than at lower densities and the oscillations are therefore far from being sinusoidal.

In the second case (the "ideal fluid EOS" in Fig. 9), we do not enforce the abiabatic condition, but allow all of the thermodynamic variables to evolve in time. As a result, the oscillations are gradually damped in time, while the star oscillates around a central density close to that of a stable star with the same rest-mass.

The rapid decrease in the oscillation amplitude is due to the dissipation of kinetic energy via shock heating. At the end of the first expansion (i.e. at the first minimum in Fig. 9), the star has expanded almost to the edge of the numerical grid. At this point, the outer parts of the initial star have formed a low-density, outer-core mantle around the highdensity inner core and the star then starts to contract. Figure 10 shows with solid lines the supersonic infall of the outer core mantle at $t=0.84 \mathrm{~ms}$, while the inner core is contracting homologously. After this "point of last good homology," the high-density inner core reaches its maximum infall velocity and then starts slowing down. The infalling low-density mantle forms a shock at the inner-core-mantle boundary (dotted lines at $t=0.98 \mathrm{~ms}$ in Fig. 10). After the inner core bounces, it expands and pressure waves at the inner-coremantle boundary feed the shock wave with kinetic energy (dashed lines at $t=1.13 \mathrm{~ms}$ in Fig. 10). In this way, the shock wave is dissipating the initial binding energy of the star so that the amplitude of the central density oscillations decreases with time. The above process is very similar to the core bounce in neutron star formation (see, for instance, the description in [50]), except for the fact that here the outer mantle is created during the first rapid expansion from material of the initial unstable star.

As a result of the damping of the radial oscillations, the star settles down, on a secular time scale, to a stable equilibrium configuration with central density somewhat smaller than the central density of a stable star with the same restmass as the initial unstable star. This is because part of the matter of the initial star forms a heated mantle around the inner core.

The evolution shown in Fig. 9 was obtained using a resolution of $96^{3}$ gridpoints. Since the initial unstable configuration is much more compact than the final configuration, the boundaries of the computational grid were placed at about $4.5 r_{s}$. As a result, the grid resolution of the initial configuration is rather low, causing an additional, non-negligible deviation of the average central rest-mass density of the pulsating star away from the expected central rest-mass density of the zero-temperature star of the same rest-mass.

The evolution of the highly nonlinear and nonadiabatic pulsations of a star when it settles down on the stable branch, underlines the importance of evolving all of the thermodynamic variables (including the specific internal energy) and the importance of using HRSC methods in order to resolve the formation and evolution of shocks correctly. These capabilities of the numerical code will be important in the correct simulation of general relativistic astrophysical events such as the merging of a neutron star binary system or the formation of a neutron star in an accretion-induced collapse of a white dwarf.

\section{Gravitational collapse of unstable configurations}

As mentioned in the previous section, the numerical scheme used in the hydrodynamical evolution is such that it causes a nonrotating relativistic star in an unstable equilibrium to expand and migrate to the configuration of the same rest-mass located on the stable branch of equilibrium configurations. In order to study the gravitational collapse to a black hole of an unstable model we need to add to the initial model a small radial perturbation in the rest-mass density distribution. A very small perturbation of the order of $\sim 1 \%$ is sufficient and its radial dependence can be simply given by $\cos \left(\pi r / 2 r_{s}\right)$, where $r$ is the coordinate distance from the center and $r_{s}$ its value at the surface of the star.

The addition of this small perturbation dominates over the truncation error and causes the star to collapse to a black hole. Note that after the perturbation is added to the initial equilibrium configuration, the constraint equations are solved 


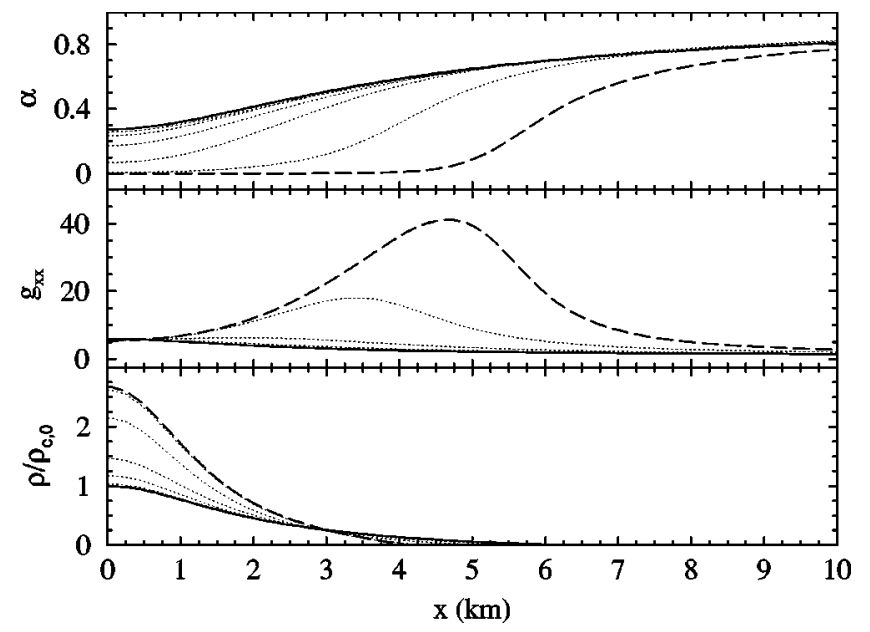

FIG. 11. Profiles along the $x$-axis of representative metric and fluid quantities during the gravitational collapse to a black hole of an unstable $N=1, \rho_{c}=8.0 \times 10^{-3}$ relativistic polytrope showing different snapshots of the time evolution. The top, medium and bottom panels show the evolution of the lapse function, of the $g_{x x}$ metric component, and of normalized rest-mass density, respectively. The thick solid and dashed lines indicate the initial and final $(t=0.29 \mathrm{~ms})$ profiles. Intermediate profiles, indicated by thin dotted dashed lines, are shown every $0.049 \mathrm{~ms}$.

to provide initial data which is a solution to the field equations [25].

The (forced) collapse to a black hole of an unstable spherical relativistic star is shown in Fig. 11 for a simulation with $128^{3}$ gridpoints in octant symmetry, using Roe's solver and an ideal fluid EOS. The figure shows the profiles along the $x$-axis of the lapse function (top panel), of the $g_{x x}$ metric component (middle panel) and of the normalized rest-mass density (bottom panel). Different lines refer to different times of the evolution, with the thick solid line in each panel indicating the initial profile and with the thick dashed line corresponding to the final time slice at $t=0.29 \mathrm{~ms}$; intermediate times (shown every $0.049 \mathrm{~ms}$ ) are indicated with dotted lines. The evolution of the lapse function shows the characteristic "collapse of the lapse," a distinctive feature of black hole formation. The evolution of the $g_{x x}$ metric component and of the rest-mass density also clearly exhibits features typical of black hole formation, such as the large peak developing in $g_{x x}$, or the continuous increase in the central rest-mass density.

While the collapse of the lapse is a good indication of the formation of a black hole, the formation of an apparent horizon (the outermost of the trapped surfaces) in the course of the simulation is an unambiguous signature of black hole formation. An apparent horizon finder based on the fast-flow algorithm [51] was used to detect the appearance of horizons, and to calculate the horizon mass. This apparent horizon finder, and its validation, is described in Ref. [52].

Figure 12 shows the evolution of the horizon mass as a function of time. Initially there is no horizon. At a time $t$ $=0.21 \mathrm{~ms}$ a black hole forms and an apparent horizon appears. As the remaining stellar material continues to accrete onto the newly formed black hole its horizon mass increases,

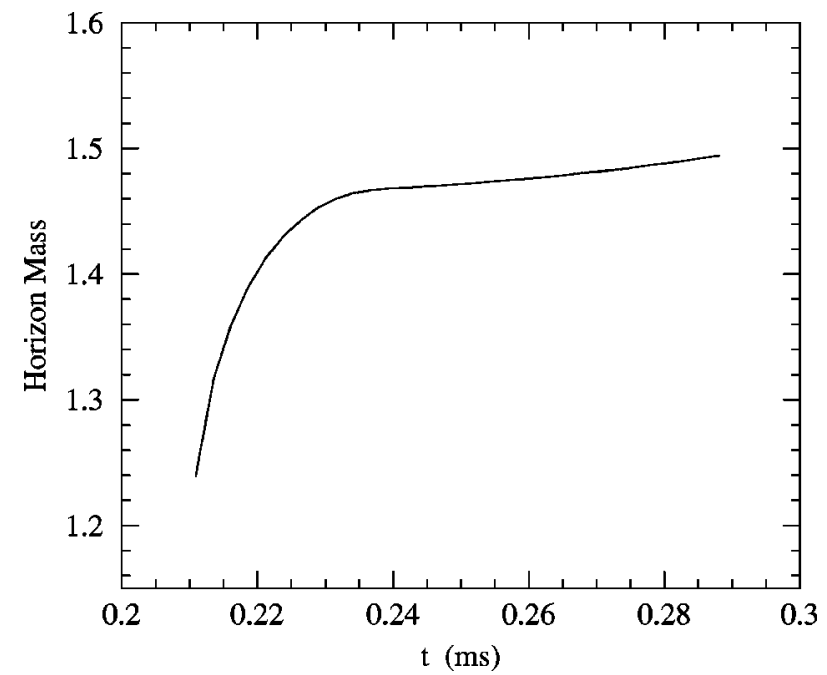

FIG. 12. Horizon mass as a function of time. A black hole is formed at $t=0.21 \mathrm{~ms}$ and the horizon mass then starts to increase as a result of accretion.

finally levelling off, until about $t=0.27 \mathrm{~ms}$. The subsequent growth of the horizon mass is the result of the increasing error due to grid stretching-the radial metric function develops a sharp peak which cannot be resolved adequately.

\section{RAPIDLY ROTATING RELATIVISTIC STARS}

\section{A. Stationary equilibrium models}

The long-term evolution of rapidly rotating, stable equilibrium relativistic stars represents a much more demanding test for a numerical code. In this case, in fact, the use of a non-zero shift vector is strictly necessary and this, in turn, involves the testing of parts of the code that are not involved in the evolution of a nonrotating stellar model. The initial data used here are numerical solutions describing general relativistic stationary and axisymmetric equilibrium models rotating uniformly with angular velocity $\Omega$. The models are constructed with the rns code $[53,54]$ (see Ref. [55] for a recent review of rotating stars in relativity) which provides the four metric potentials $\nu, B, \mu$, and $\omega$ needed to describe the spacetime with line element

$$
\begin{aligned}
d s^{2}= & -e^{2 \nu} d t^{2}+B^{2} e^{-2 \nu} r^{2} \sin ^{2} \theta(d \phi-\omega d t)^{2} \\
& +e^{2 \mu}\left(d r^{2}+r^{2} d \theta^{2}\right) .
\end{aligned}
$$

In the nonrotating limit, the above metric reduces to the metric of a static, spherically symmetric spacetime in isotropic coordinates. A rotating model is uniquely determined upon specification of the EOS and two parameters, such as the central rest-mass density and the ratio of the polar to the equatorial coordinate radii (axes ratio).

Using the standard Jacobian transformations between the spherical polar coordinates $(r, \theta, \phi)$ and the Cartesian coordinates $(x, y, z)$, the initial data for a rotating star are transformed to Cartesian coordinates. Convergence tests of the initial data on the Cartesian grid at various resolutions, show that the Hamiltonian and momentum constraints converge at 
second-order everywhere except at the surface of the star, where some high-frequency noise is present. This noise is due to Gibbs phenomena at the surface of the star, which are inherent to the method [56] used in the construction of the 2D initial data (see the relevant discussion in Ref. [54]). To our knowledge, all currently available methods for constructing initial data describing rotating relativistic stars suffer from some kind of Gibbs phenomena at the surface of the star, with the only exception being a recent multi-domain spectral method that uses surface-adapted coordinates [57]. The high-frequency noise does not appear to affect the longterm evolution of the initial data at the grid resolutions employed in our simulations. The evolution is carried out up to several rotational periods, using the shift 3-vector obtained from the solution of the stationary problem, which we do not evolve in time.

We have evolved models at various rotation rates and for several polytropic EOS, all showing similar long-term behavior and convergence. Hereafter we will focus on an $N$ $=1$ polytropic model, rotating at $92 \%$ of the allowed massshedding limit for a uniformly rotating star with the same central rest-mass density. In particular, we have chosen a stellar model with the same central rest-mass density as the nonrotating model of Sec. IV A and which is significantly flattened by the rapid rotation (the polar coordinate radius is only $70 \%$ of the equatorial coordinate radius).

Similarly to what is observed in the numerical evolution of nonrotating stars, the truncation errors trigger, in a rapidly rotating star, oscillations that are quasi-radial. As a result, the rotating star pulsates mainly in its fundamental quasi-radial mode and, during the long-term evolution, its central restmass density drifts towards higher values. Also in this case, both the amplitude of the pulsations and the central density growth rate converge to zero at nearly second order with increasing grid resolution.

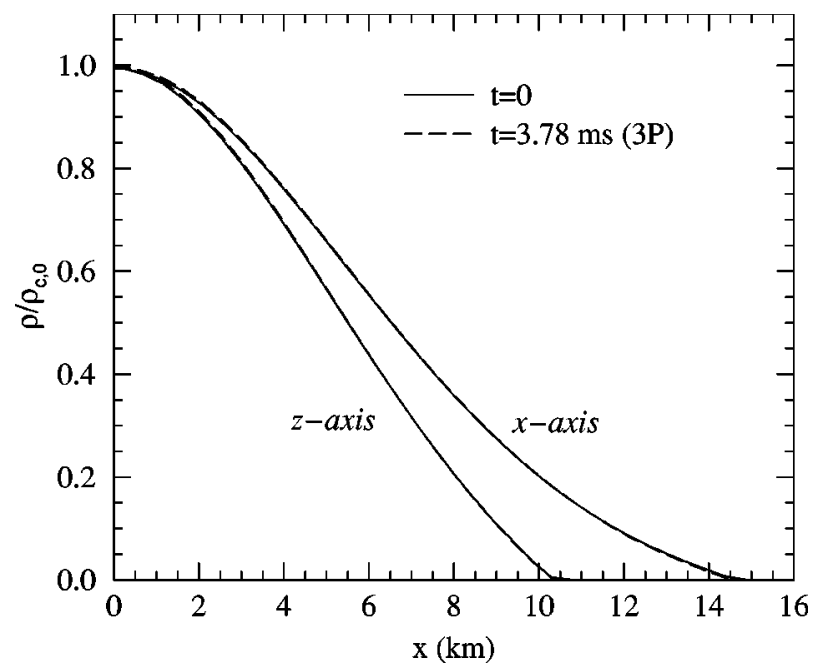

FIG. 13. Profiles of the (normalized) rest-mass density along the $x$-axis and $z$-axis at two coordinate times, $t=0$ (solid lines) and $t$ $=3.78 \mathrm{~ms}$ (dashed lines), corresponding to three rotational periods $(P)$. The star is an $N=1, \rho_{c}=1.28 \times 10^{-3}$ polytrope rotating at $92 \%$ of the mass-shedding limit. The simulation has been performed only in the volume above the $(x, y)$ plane which is covered with $129 \times 129 \times 66$ gridpoints.

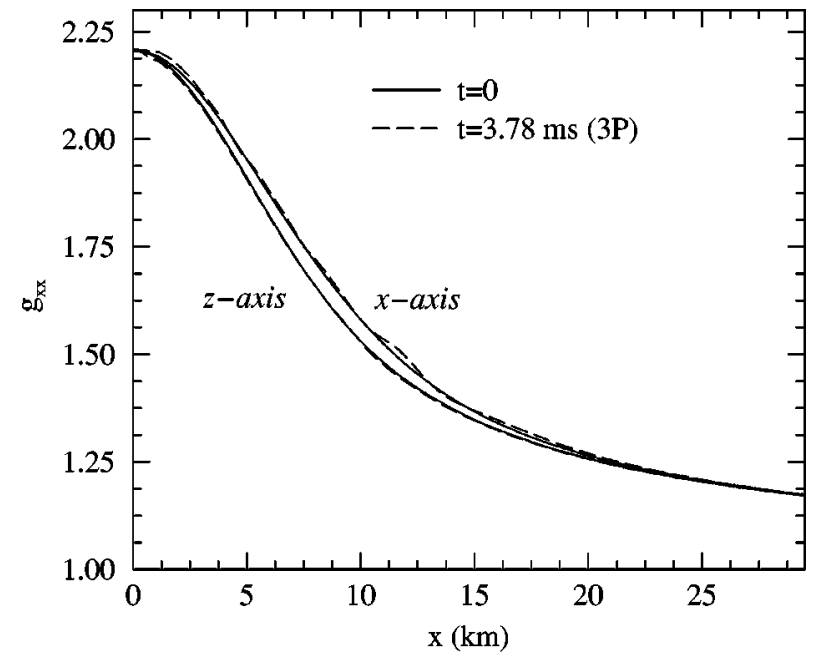

FIG. 14. Profile of the metric component $g_{x x}$ along the $x$-axis and $z$-axis at two different coordinate times, for the same evolution shown in Fig. 13.

Our simulations have been performed only in the volume above the $(x, y)$ plane which is covered with $129 \times 129 \times 66$ gridpoints. At such grid resolutions, we have been able to evolve a stationary rapidly rotating relativistic star for three complete rotational periods, before the numerical solution departs noticeably from the initial configuration. Note that much longer evolution times (more than an order of magnitude longer and essentially limited by the time available) can be achieved if the spacetime is held fixed and only the hydrodynamical equations in a curved background are evolved. This has been demonstrated recently in Ref. [17], with a code based on the one used in the present paper and in which a third-order piecewise parabolic method (PPM) [58] was used for the hydrodynamical evolution and applied to the study of nonlinear $r$-modes in rapidly rotating relativistic stars and the occurrence of differential of a kinematical differential rotation [59] (see Refs. [60,61] for a recent review on the $r$-mode instability). While our current second-order

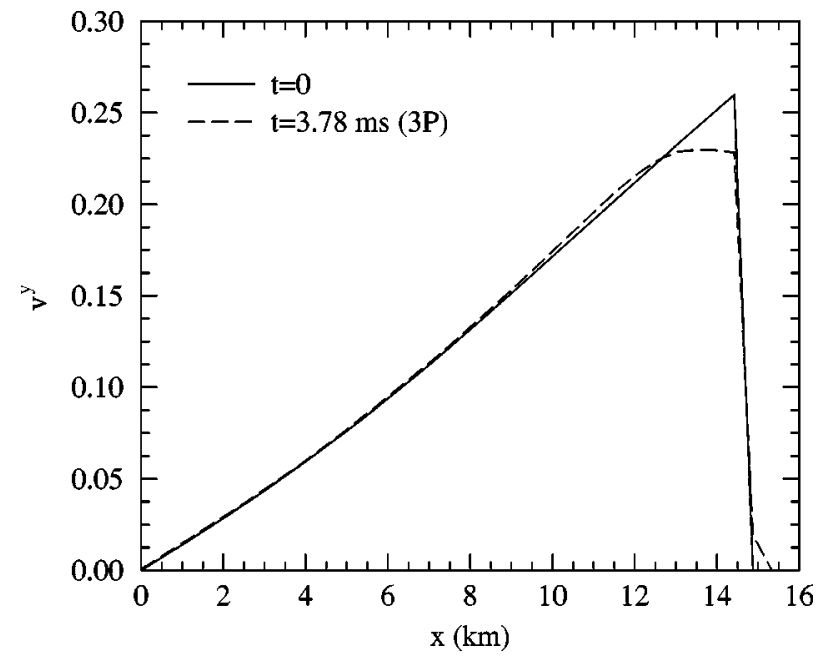

FIG. 15. The velocity component $v^{y}$ along the $x$-axis at two different coordinate times, for the same evolution as in Fig. 13. 
TVD method with the MC limiter is not as accurate (for the same grid resolution) as the third-order PPM method, it has, nevertheless, a very good accuracy, significantly better than that of the minmod limiter.

Results of our simulations of rapidly-rotating stars are plotted in Figs. 13-15. In particular, Fig. 13 shows the (normalized) rest-mass density along the $x$ and $z$ axes at two coordinate times, $t=0$ (solid lines) and $t=3.78 \mathrm{~ms}$ (dashed lines), with the latter corresponding to three rotational periods. The outer boundary of the grid is placed at about twice the equatorial radius. After three rotational periods, the restmass density profile is still very close to the initial one. Similarly, Fig. 14 shows the metric component $g_{x x}$ along the $x$ and $z$ axes at the same coordinate times of Fig. 13. Again, the change in $g_{x x}$ is minimal and only near the stellar surface can one observe a noticeable difference [the error there grows faster for rotating stars (than for nonrotating stars), due to the Gibbs phenomenon in the initial data].

In addition to triggering the appearance of quasiradial pulsations and the secular increase in the central rest-mass density, the truncation errors also induce the formation of a local maximum at the stellar surface for the evolved "momentum" variable $\widetilde{S}_{j}$ [cf. Eq. (7)]. The existence of this local extremum reduces, at the surface of the rotating star, the order of our TVD schemes to first-order only. As a result, the angular momentum profile at the surface gradually drifts away from the initial uniformly rotating one, with the rate of convergence of this drift being only first-order with increasing grid resolution. We emphasize, however, that this is only a local effect: everywhere else inside the star, the angular momentum evolution is second-order accurate. Figure 15 shows the velocity component $v^{y}$ along the $x$-axis at the same coordinate times of Figs. 13 and 14. Alternative evolution schemes based on third-order methods have been shown to have a smaller truncation error at the surface of the star, both for 2D and 3D evolutions of the same initial data [43,17], at least in the Cowling approximation.

Note that plotting the velocity profile as in Fig. 15 allows one to ascertain the accuracy in the preservation of the velocity field. Isocontours or vector plots of the velocity field can, in fact, easily mask the secular evolution shown in Fig. 15. We also note that the variable evolved in the code is not the rotational velocity, but a corresponding momentum component which depends on the local rest-mass [cf. Eq. (7)]. The error in the rotational velocity near the surface is therefore also influenced by the small value of the rest-mass density in that region.

\section{B. Quasi-radial modes of rapidly rotating relativistic stars}

The quasi-radial pulsations of rotating neutron stars are a potential source of detectable gravitational waves and could be excited in various astrophysical scenarios, such as a rotating core-collapse, a core-quake in a rotating neutron star (due to a large phase-transition in the equation of state) or the formation of a high-mass neutron star in a binary neutron star merger. An observational detection of such pulsations would yield valuable information about the equation of state of relativistic stars [62]. So far, however, the quasi-radial
TABLE III. Comparison of small-amplitude quasi-radial pulsation frequencies obtained with the present 3D code in fixed spacetime, with frequencies obtained with an independent $2 \mathrm{D}$ code. The equilibrium model is an $N=1$ relativistic polytrope rotating at $92 \%$ of the mass-shedding limit.

\begin{tabular}{cccc}
\hline \hline Mode & $\begin{array}{c}\text { Present 3D code } \\
(\mathrm{kHz})\end{array}$ & $\begin{array}{c}2 \mathrm{D} \text { code } \\
(\mathrm{kHz})\end{array}$ & $\begin{array}{c}\text { Relative Difference } \\
(\%)\end{array}$ \\
\hline$F$ & 2.468 & 2.456 & 0.5 \\
$H 1$ & 4.344 & 4.357 & 0.3 \\
$H 2$ & 6.250 & 6.270 & 0.3 \\
\hline \hline
\end{tabular}

modes of rotating relativistic stars have been studied only under simplifying assumptions such as in the slow-rotation approximation $[63,64]$ or in the relativistic Cowling approximation $[48,65]$. The spectrum of quasi-radial pulsations in full general relativity has not been solved to date with perturbation techniques (see Ref. [55] for a recent review of the subject).

In this section we take a step forward in the solution of this long standing problem in the physics of relativistics stars and obtain the first mode-frequencies of rotating stars in full general relativity and rapid rotation. As done in Sec. IV B for the radial pulsation of nonrotating stars, we take advantage of the very small numerical viscosity of our code to extract physically relevant information from the quasi-radial perturbations induced by truncation errors. The ability to do so demonstrates that our current numerical methods are mature enough to obtain answers to new problems in the physics of relativistics stars.

Following the approach outlined in Sec. IV B, we have first computed the quasi-radial mode frequencies from numerical evolutions of the GR-Hydro equations in a fixed spacetime evolution in order to compare with recent results coming from an independent 2D nonlinear evolution code [65]. Table III shows the comparison of between the eigenfrequencies computed in the Cowling approximation with the 2D code for the equilibrium model of the previous section. Note that the newly obtained frequencies differ by less than $0.5 \%$, verifying that our code can accurately reproduce them.

Next, we have computed the quasi-radial frequencies in coupled hydrodynamical and spacetime evolutions for rapidly rotating stars. As mentioned before, this is a novel study and the results obtained cannot be compared with corresponding results in the literature. To study this, we have carried out two types of analysis. Firstly, we have followed the same procedure used in the case of a nonrotating star and obtained the normalized frequency spectrum of oscillations induced by the truncation errors. Secondly, we have computed the frequency spectrum of oscillations triggered by a small but specified perturbation. More precisely, we have introduced the same radial perturbation in the rest-mass density used in Sec. IV D to induce collapse: i.e. $A \cos \left(\pi r / 2 r_{p}\right)$, where $A=0.02, r$ is the coordinate distance from the center, and $r_{p}$ is the radial coordinate position of the poles. When compared, the results of the two treatments indicate that the fundamental mode frequency agrees to within $2 \%$, while the H1 mode near the mass-shedding limit is probably accurate 
TABLE IV. Quasi-radial pulsation frequencies for a sequence of rotating $N=1$ polytropes with rotation rates up to $97 \%$ of the massshedding limit. The frequencies of the fundamental mode $F$ and of the first overtone $H 1$ are computed from coupled hydrodynamical and spacetime evolutions. The ratio of polar $r_{p}$ to equatorial $r_{e}$ coordinate radii of the rotating models is also shown.

\begin{tabular}{cccc}
\hline \hline$r_{p} / r_{e}$ & $\Omega / \Omega_{K}$ & $F(\mathrm{kHz})$ & $H 1(\mathrm{kHz})$ \\
\hline 1.000 & 0.000 & 1.450 & 3.958 \\
0.950 & 0.407 & 1.411 & 3.852 \\
0.850 & 0.692 & 1.350 & 3.867 \\
0.825 & 0.789 & 1.329 & 3.894 \\
0.775 & 0.830 & 1.287 & 3.953 \\
0.750 & 0.867 & 1.265 & 4.031 \\
0.725 & 0.899 & 1.245 & 3.974 \\
0.700 & 0.929 & 1.247 & 3.887 \\
0.675 & 0.953 & 1.209 & 3.874 \\
0.650 & 0.974 & 1.195 & 3.717 \\
\hline \hline
\end{tabular}

to several percent only (at this resolution).

To study quasi-radial modes of rapidly rotating relativistic stars we have built a sequence of models having the same grid resolution, the same equation of state and central restmass density used in the previous section, varying only the rotation rate $\Omega$. The sequence starts with a nonrotating star and terminates with a star at $97 \%$ of the maximum allowed rotational frequency $\Omega_{K}=0.5363 \times 10^{4} \mathrm{~s}^{-1}$ for uniformly rotating stars (mass-shedding limit). The results of these simulations are reported in Table IV and shown in Fig. 16, where the frequencies of the lowest two quasi-radial modes are shown. Interestingly, the fundamental mode-frequencies (solid lines) and their first overtones (dashed lines) show a

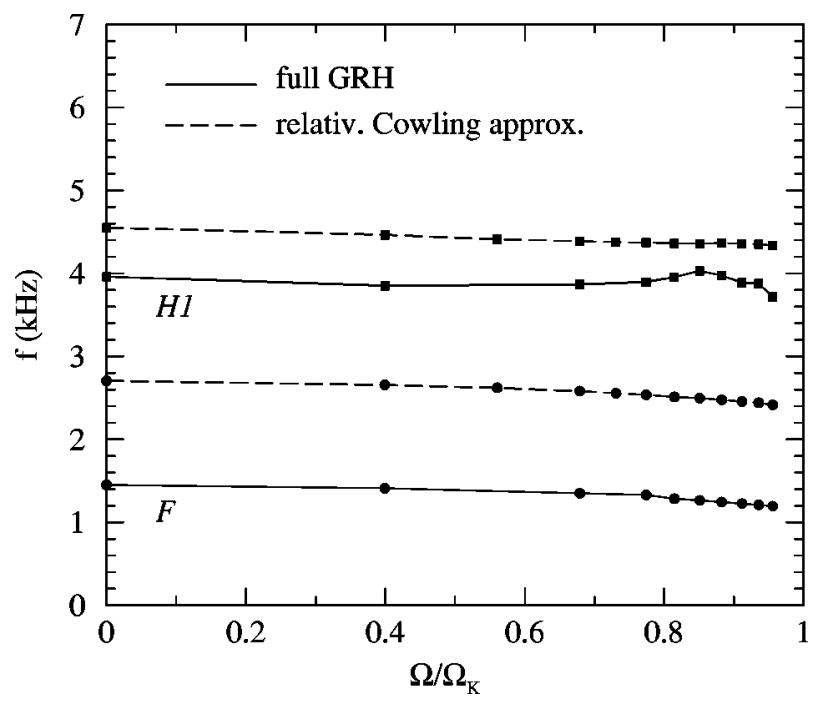

FIG. 16. Quasi-radial pulsation frequencies for a sequence of rotating $N=1$ polytropes and a number of different rotation rates. The frequencies of the fundamental mode $F$ (filled squares) and of the first overtone $H 1$ (filled circles) are computed from coupled hydrodynamical and spacetime evolutions (solid lines). The sequences are also compared with the corresponding results obtained from computations in the relativistic Cowling approximation. dependence on the increased rotation which is similar to the one observed for the corresponding frequencies in the Cowling approximation [65].

In particular, the $F$-mode frequency decreases monotonically as the maximum rotation rate is approached. Near the mass-shedding limit, the frequency is $18 \%$ smaller than the frequency of the nonrotating star. The difference between the $F$-mode frequency computed here and the corresponding result in the Cowling approximation is nearly constant. Thus, one can construct an approximate empirical relation for the fundamental quasi-radial frequency of rapidly rotating stars, using only the corresponding frequency in the Cowling approximation, $F_{\text {Cowling }}$ and the frequency of the fundamental radial mode in the nonrotating limit, $F_{\Omega=0}$. For the particular sequence shown above, the empirical relation reads

$$
F=\left(F_{\text {Cowling }}-1.246\right) \mathrm{kHz},
$$

and yields the correct frequencies with an accuracy of better than $2 \%$ for the most rapidly rotating model. More generally, if $F_{\text {Cowling }, \Omega=0}$ is the frequency of the fundamental radial mode in the Cowling approximation, then the empirical relation can be written as

$$
F=F_{\Omega=0}+F_{\text {Cowling }}-F_{\text {Cowling }, \Omega=0} .
$$

Such an empirical relation is very useful, as it allows one to obtain a good estimate of the fundamental quasi-radial mode frequency of rapidly rotating stars by solving the hydrodynamical problem in a fixed spacetime, rather than solving the much more expensive evolution problem in which the spacetime and the hydrodynamics are coupled.

The frequency of the $H 1$ mode shows a non-monotonic decrease as the mass-shedding limit is approached, departing from the behavior obtained in the Cowling approximation. The oscillations in the frequency at larger rotation rates could be due to "avoided crossings" with frequencies of other modes of oscillation. (We recall that "avoided crossing" refers to the typical behavior shown by two eigenfrequency curves which approach smoothly but then depart from each other without crossing. At the point of closest approach, the properties of the modes on each sequence are exchanged [66]). Similar avoided crossings have been observed also in the Cowling approximation for higher overtones and near the mass-shedding limit (see Refs. [48,65]). Our results indicate therefore that the avoided crossings in a sequence of relativistic rotating stars occur for smaller rotation rates than predicted by the Cowling approximation. This increases the importance of avoided crossings and makes the frequency spectrum in rapidly rotating stars more complex than previously thought.

\section{GRAVITATIONAL WAVES FROM A PULSATING STAR}

The ability to extract gravitational wave information from simulations of relativistic compact objects is an important feature of any 3D general relativistic hydrodynamics code. To assess the ability of our code to extract self-consistent and accurate gravitational waveforms we have excited simple quadrupolar perturbations in our standard spherical $N=1$ 


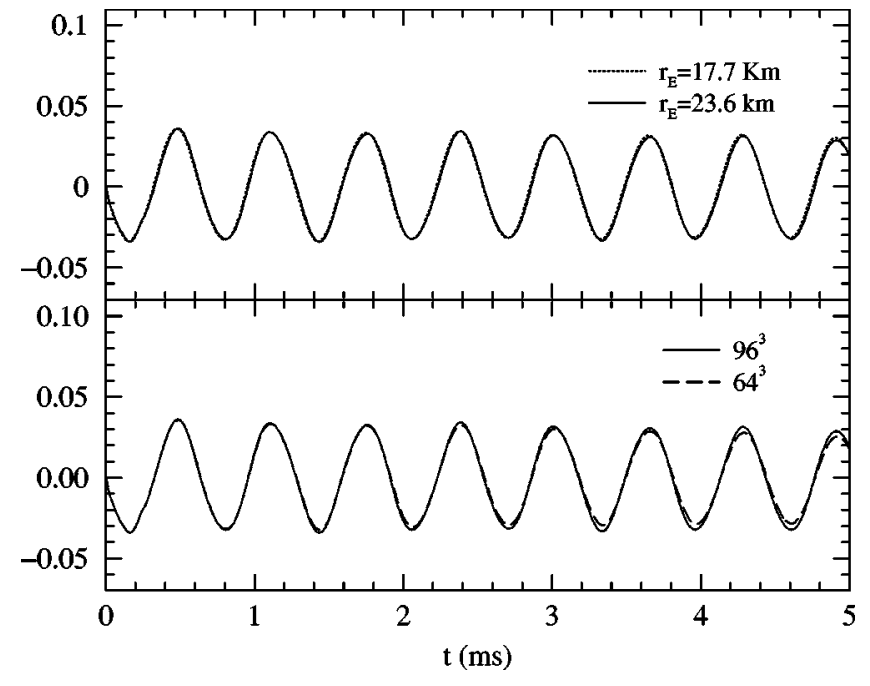

FIG. 17. Gravitation-wave extraction $\left(\psi^{(20)}\right)$ of a perturbed nonrotating relativistic star, pulsating mainly in the fundamental quadrupolar mode. The top panel shows the rescaled waverforms as extracted at $r_{\mathrm{E}}=17.7 \mathrm{~km}$ (dotted line) and at $r_{\mathrm{E}}=23.6 \mathrm{~km}$ (solid line). The lower panel, on the other hand, shows the amplitude of $\psi^{20}$ extracted at $r_{\mathrm{E}}=23.6 \mathrm{~km}$ for two different resolutions of $64^{3}$ (dashed line) and $96^{3}$ gridpoints (solid line), respectively.

polytrope. In particular, on the basis of the angular behavior of the $l=2, f$-mode in linear perturbation theory, we have introduced in the initial model a perturbation in the velocity of the form

$$
u_{\theta}(t=0)=A \sin \left(\pi r / r_{s}\right) \sin \theta \cos \theta
$$

where $A=0.02$ is the amplitude of the perturbation and $r_{s}$ is the coordinate radius of the star.

Following York [25], we have then constructed the initial data for the perturbed model by solving the constraint equations for the unperturbed model with added perturbations and then proceeded to evolve this solution in time. As a response to the initial perturbations, the star has started a series of periodic oscillations, mainly in the fundamental quadrupolar mode of oscillation. Other, higher-order modes are also excited (and observed) but these are several orders of magnitude smaller and play no dynamical role.

As a consequence of the time-varying mass quadrupolar triggered by the oscillations, the perturbed star emits gravitational waves, which are extracted through a perturbative technique discussed in detail in Refs. [67-69], and in which the Zerilli function is expanded in terms of spherical harmonics with each component being the solution of an ordinary differential equation.

We plot, in Fig. 17, the $l=2, m=0$ component of the Zerilli function $\psi^{20}$. The upper panel, in particular, shows the waverforms as extracted at $r_{\mathrm{E}}=17.7 \mathrm{~km}$ (dotted line) and at $r_{\mathrm{E}}=23.6 \mathrm{~km}$ (solid line) respectively, with the first having been rescaled as $r^{-3 / 2}$ to allow a comparison. The very good agreement between the two waveforms is an indication that the gravitational waves have reached their asymptotic waveform. The lower panel, on the other hand, shows the amplitude of $\psi^{20}$ extracted at $r_{\mathrm{E}}=23.6 \mathrm{~km}$ for two different reso- lutions of $64^{3}$ (dashed line) and $96^{3}$ gridpoints (solid line), respectively. Note that the gravitational wave signal converges to a constant amplitude, as the true gravitational-wave damping timescale for this mode is several orders of magnitude larger than the total evolution time shown. The small decrease in the amplitude observed in our numerical evolution is thus entirely due to the effective numerical viscosity of our scheme. At a resolution of $96^{3}$ gridpoints, the effective numerical viscosity is sufficiently low to allow for a quantitative study of gravitational waves from pulsating stars over a time scale of many dynamical times (the largest relative numerical error estimated on the basis of the simulations presented in Fig. 17 is $3.3 \%$ ).

As done in the previous sections, we have compared the frequencies derived from our numerical simulations with those obtained from perturbative techniques for $f$-mode oscillations of $N=1$ polytropes. Again in this case the comparison has revealed a very good agreement between the two approaches. As one would expect, the dominant frequency of the gravitational waves we extract $(1.56 \mathrm{kHz})$ agrees with the fundamental quadrupole $f$-mode frequency of the star $(1.58$ $\mathrm{kHz}$ ) [47] to within $1.3 \%$ (at a resolution of $96^{3}$ grid-points).

\section{CONCLUSIONS}

We have presented results obtained with a $3 \mathrm{D}$ general relativistic code GR_ASTRO in a comprehensive study of the long-term dynamics of relativistic stars. The code has been built by the Washington University/Albert Einstein Institute collaboration for the NASA Neutron Star Grand Challenge Project [12] and is based on the Cactus Computational Toolkit [13]. The simulations reported here have benefited from several new numerical strategies that have been implemented in the code and that concern both the evolution of the field equations and the solution of the hydrodynamical equations. In addition to the features of the code discussed in paper I, the present version of the code can construct various types of initial data representing spherical and rapidly rotating relativistic stars, extract gravitational waves produced during the simulations and track the presence of an apparent horizon when formed.

All of these improvements have allowed tests and performances well superior to those reported in the companion paper I. With this improved setup we have shown that our code is able to succesfully pass stringent long-term evolution tests, such as the evolution of both, static and rapidly rotating, stationary configurations. We have also considered the evolution of relativistic stars unstable to either gravitational collapse or expansion. In particular, we have shown that unstable relativistic stars can, in the course of a numerical evolution, expand and migrate to the stable branch of equilibrium configurations. As an application of this property, we have studied the large-amplitude nonlinear pulsations produced by the migration. Nonlinear oscillations are expected to accompany the formation of a proto-neutron star after a supernova core-collapse or after an accretion-induced collapse of a white dwarf.

Particularly significant for their astrophysical application, we have investigated the pulsations of both rapidly rotating 
and nonrotating relativistic stars and compared the computed frequencies of radial, quasi-radial and quadrupolar oscillations with the frequencies obtained from perturbative methods or from axisymmetric nonlinear evolutions. We have shown that our code reproduces these results with excellent accuracy. As a particularly relevant result, we have obtained the first mode-frequencies of rotating stars in full general relativity and rapid rotation. A long standing problem, such frequencies had not been obtained so far by other methods.

In our view the results discussed in this paper have a double significance. Firstly, they establish the accuracy and reliability of the numerical techniques employed in our code, which, to the best of our knowledge, represent the most accurate long-term 3D evolutions of relativistic stars available to date. Secondly, they show that our current numerical methods are mature enough to obtain answers to new problems in the physics of relativistic stars.

\section{ACKNOWLEDGMENTS}

It is a pleasure to thank K.D. Kokkotas for many discussions and for providing us with the linear perturbation frequencies. We have also benefited from many discussions with M. Alcubierre, S. Bonazzola, B. Brügman, J.M. Ibánez,
J. Miller, M. Shibata, K. Uryu, and Shin Yoshida. The simulations in this paper have made use of code components developed by several authors. In what follows we report the names of the different components, their use and their main author. BAM (elliptic equation solver), B. Brügman; AHFINDER (apparent horizon finder), M. Alcubierre; CONFADM (evolution scheme for the field equations) and MAHC (evolution scheme for the GRHydro equations), M. Miller; PRIM-SOL (solver for the hydrodynamical primitive variables), P. Gressman; RNS-ID (initial data solver for rotating and perturbed relativistic stars), N. Stergioulas; EXTRACT (gravitational wave analysis), G. Allen. The application code is built on the CACTUS Computational Toolkit written by P. Walker et al. (version 3) and T. Goodale et al. (version 4). Financial support for this research has been provided by the NSF KDI Astrophysics Simulation Collaboratory (ASC) project (Phy 99-79985), NASA Earth and Space Science Neutron Star Grand Challenge Project (NCCS-153), NSF NRAC Project Computational General Relativistic Astrophysics (93S025), and the EU Programme "Improving the Human Research Potential and the Socio-Economic Knowledge Base" (Research Training Network Contract HPRNCT-2000-00137).
[1] For a summary of the present and planned high energy missions, see heasarc.gsfc.nasa.gov/docs/heasarc/missions.html

[2] K. Thorne, in Proceedings of the Eight Nishinomiya-Yukawa Symposium on Relativistic Cosmology, edited by M. Sasaki (Universal Academy Press, Japan, 1994).

[3] J.R. Wilson, G.J. Mathews, and P. Marronetti. Phys. Rev. D 54, 1317 (1996).

[4] T. Nakamura and K. Oohara, invited talk at "Numerical Astrophysics 1998," gr-qc/9812054.

[5] T.W. Baumgarte, S.A. Hughes, and S.L. Shapiro, Phys. Rev. D 60, 087501 (1999)

[6] J.A. Font, M. Miller, W.M. Suen, and M. Tobias, Phys. Rev. D 61, 044011 (2000).

[7] M. Miller, W.-M. Suen, and M. Tobias, Phys. Rev. D 63, 121501(R) (2001).

[8] M. Shibata, Phys. Rev. D 60, 104052 (1999).

[9] M. Shibata and K. Uryu, Phys. Rev. D 61, 064001 (2000).

[10] M. Shibata, T.W. Baumgarte, and S.L. Shapiro, Phys. Rev. D 61, 044012 (2000).

[11] The NASA Grand Challenge Project is described at http:// wugrav.wustl.edu/Relativ/nsgc.html

[12] The code and its documentation can be found at wugrav.wustl.edu/Codes/GR3D

[13] For information see www.cactuscode.org

[14] R. Arnowitt, S. Deser, and C.W. Misner, in Gravitation: An Introduction to Current Research, edited by L. Witten (Wiley, New York, 1962), p. 227.

[15] C. Bona, J. Massó, E. Seidel, and J. Stela, Phys. Rev. D 56, 3405 (1997).

[16] M. Alcubierre, W. Benger, B. Brügmann, G. Lanfermann, L. Nerger, E. Seidel, and R. Takahashi, Phys. Rev. Lett. 87, 271103 (2001).
[17] N. Stergioulas and J.A. Font, Phys. Rev. Lett. 86, 1148 (2001).

[18] E. Seidel and W.-M. Suen, Phys. Rev. D 42, 384 (1990).

[19] T. Zwerger and E. Muller, Astron. Astrophys. 320, 209 (1997).

[20] H. Dimmelmeier, J.A. Font, and E. Mueller, Astrophys. J. Lett. 560, L163 (2001).

[21] M. Shibata and T. Nakamura, Phys. Rev. D 52, 5428 (1995).

[22] T.W. Baumgarte and S.L. Shapiro, Phys. Rev. D 59, 024007 (1999).

[23] M. Alcubierre, B. Brügmann, T. Dramlitsch, J.A. Font, P. Papadopoulos, E. Seidel, N. Stergioulas, W.-M. Suen, and R. Takahashi, Phys. Rev. D 62, 044034 (2000).

[24] R.D. Richtmyer and K.W. Morton, Difference Methods for Initial Value Problems (Interscience Publishers, New York, 1967).

[25] J. York, in Sources of Gravitational Radiation, edited by L. Smarr (Cambridge University Press, Cambridge, England, 1979).

[26] H. Friedrich (private communication).

[27] M. Alcubierre, G. Allen, B. Brügmann, E. Seidel, and W.-M. Suen, Phys. Rev. D 62, 124011 (2000).

[28] J. Balakrishna, G. Daues, E. Seidel, W.-M. Suen, M. Tobias, and E. Wang, Class. Quantum Grav. 13, L135 (1996).

[29] F. Banyuls, J.A. Font, J.M. Ibánez, J.M. Martí, and J.A. Miralles, Astrophys. J. 476, 221 (1997).

[30] C. Bona, J. Massó, E. Seidel, and J. Stela, Phys. Rev. Lett. 75, 600 (1995).

[31] M. Alcubierre, Phys. Rev. D 55, 5981 (1997).

[32] M. Alcubierre and J. Massó, Phys. Rev. D 57, 4511 (1998).

[33] S. Teukolsky, Phys. Rev. D 61, 087501 (2000).

[34] J.M. Ibánez, M.A. Aloy, J.A. Font, J.M. Martí, J.A. Miralles, and J.A. Pons, in Godunov Methods: Theory and Applications, edited by E.F. Toro (Kluwer Academic Plenum, Dordrecht, 2001). 
[35] J.M. Ibánez and J.M. Martí, J. Comput. Appl. Math. 109, 173 (1999).

[36] J.A. Font, Living Rev. Relativ. 3, 2 (2000); http:/// www.livingreviews.org/Articles/Volume2/2000-2font/

[37] C. Hirsch, Numerical Computation of Internal and External Flows (Wiley-Interscience, New York, 1992).

[38] P.L. Roe, J. Comput. Phys. 43, 357 (1981).

[39] R. Donat and A. Marquina, J. Comput. Phys. 125, 42 (1996).

[40] A. Harten, SIAM (Soc. Ind. Appl. Math.) J. Numer. Anal. 21, 1 (1984).

[41] B.J. van Leer, J. Comput. Phys. 23, 276 (1977).

[42] N. Stergioulas, J.A. Font, and K.D. Kokkotas, in Proceedings of the 19th Texas Symposium on Relativistic Astrophysics, edited by É. Aubourg et al. [Nucl. Phys. B (Proc. Suppl.) 80, 233 (2000)].

[43] J.A. Font, N. Stergioulas, and K.D. Kokkotas, Mon. Not. R. Astron. Soc. 313, 678 (2000).

[44] T.W. Baumgarte, S.A. Hughes, L. Rezzolla, S.L. Shapiro, and M. Shibata, in General Relativity and Relativistic Astrophysics-Eighth Canadian Conference, edited by C.P. Burgess and R.C. Myers, AIP Conf. Proc. No. 493 (AIP, Melville, NY, 1999), p. 53.

[45] K.D. Kokkotas and B.G. Schmidt, Living Rev. Relativ. 2, 2 (1999); http:///www.livingreviews.org/Articles/Volume2/19992kokkotas/

[46] P.N. McDermott, H.M. Van Horn, and J.F. Sholl, Astrophys. J. 268, 837 (2001).

[47] K.D. Kokkotas (private communication).

[48] S. Yoshida and Y. Eriguchi, Mon. Not. R. Astron. Soc. 322, 389 (2001).

[49] S.H. Hawley and M.W. Choptuik, Phys. Rev. D 62, 104024 (2000).

[50] R. Moenchmeyer, G. Schaefer, E. Mueller, and R.E. Kates, Astron. Astrophys. 246, 417 (1991).

[51] C. Gundlach, Phys. Rev. D 57, 863 (1998).

[52] M. Alcubierre, S. Brandt, B. Brügmann, C. Gundlach, J.
Massó, and P. Walker, Class. Quantum Grav. 17, 2159 (2000).

[53] N. Stergioulas and J.L. Friedman, Astrophys. J. 444, 306 (1995).

[54] T. Nozawa, N. Stergioulas, E. Gourgoulhon, and Y. Eriguchi, Astron. Astrophys., Suppl. Ser. 132, 431 (1998).

[55] N. Stergioulas, Living Rev. Relativ. 1, 8 (1998); http:/// www.livingreviews.org/Articles/Volume1/1998-8stergio/

[56] H. Komatsu, Y. Eriguchi, and I. Hachisu, Mon. Not. R. Astron. Soc. 237, 355 (1989).

[57] S. Bonazzola, E. Gourgoulhon, and J.-A. Marck, Phys. Rev. D 58, 104020 (1998).

[58] P. Collela and P.R. Woodward, J. Comput. Phys. 54, 174 (1984).

[59] L. Rezzolla, F.K. Lamb, and S.L. Shapiro, Astrophys. J. Lett. 531, L139 (2000); L. Rezzolla, F.K. Lamb, D. Markovic, and S.L. Shapiro, Phys. Rev. D 64, 104013 (2001).

[60] N. Andersson and K.D. Kokkotas, Int. J. Mod. Phys. D 10, 381 (2001).

[61] J.L. Friedman and K.H. Lockitch, in Proceedings of the 9th Marcel Grossman Meeting, edited by V. Gurzadyan, R. Jantzen, and R. Ruffini (World Scientific, Singapore, 2001).

[62] K.D. Kokkotas, N. Andersson, and T.A. Apostolatos, Mon. Not. R. Astron. Soc. 320, 307 (2001).

[63] J.B. Hartle and J.L. Friedman, Astrophys. J. 196, 653 (1975).

[64] B. Datta, S.S. Hasan, P.K. Sahu, and A.R. Prasanna, Int. J. Mod. Phys. D 7, 49 (1998).

[65] J.A. Font, H. Dimmelmeier, A. Gupta, and N. Stergioulas, Mon. Not. R. Astron. Soc. 325, 1463 (2001).

[66] W. Unno, Y. Osaki, H. Ando, H. Saio, and H. Shibahashi, Nonradial Oscillation of Stars, 2nd ed. (University of Tokyo Press, Tokyo, 1989).

[67] K. Camarda and E. Seidel, Phys. Rev. D 59, 064026 (1999).

[68] M.E. Rupright, A.M. Abrahams, and L. Rezzolla, Phys. Rev. D 58, 044005 (1998).

[69] L. Rezzolla, A.M. Abrahams, R. Matzner, M. Rupright, and S. Shapiro, Phys. Rev. D 59, 064001 (1999). 